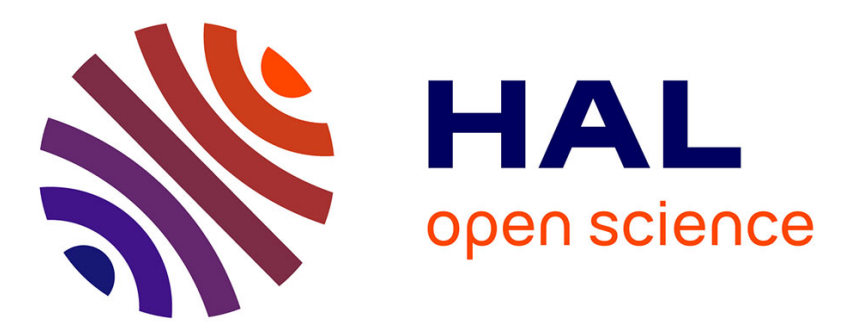

\title{
On the non-thermal nature of distributions of electrons accelerated by high intensity lasers at the vacuum-plasma interface
}

Stefan Hüller, Anna Porzio, Jean-Claude Adam, Anne Héron

\section{- To cite this version:}

Stefan Hüller, Anna Porzio, Jean-Claude Adam, Anne Héron. On the non-thermal nature of distributions of electrons accelerated by high intensity lasers at the vacuum-plasma interface. Physics of Plasmas, 2019, 26, pp.083107. 10.1063/1.5111934 . hal-02186783

\section{HAL Id: hal-02186783 \\ https://hal.science/hal-02186783}

Submitted on 17 Jul 2019

HAL is a multi-disciplinary open access archive for the deposit and dissemination of scientific research documents, whether they are published or not. The documents may come from teaching and research institutions in France or abroad, or from public or private research centers.
L'archive ouverte pluridisciplinaire HAL, est destinée au dépôt et à la diffusion de documents scientifiques de niveau recherche, publiés ou non, émanant des établissements d'enseignement et de recherche français ou étrangers, des laboratoires publics ou privés. 
On the non-thermal nature of distributions of electrons accelerated by high intensity lasers at the vacuum-plasma interface a)

S. Hüller, ${ }^{1}$ A. Porzio, ${ }^{2,1}$ J.-C. Adam, ${ }^{1}$ and A. Héron ${ }^{1}$

${ }^{1)}$ Centre de Physique Théorique(CPHT), CNRS, Ecole Polytechnique, IP Paris, 91128 Palaiseau Cedex, France

${ }^{2)}$ LAGA, Institut Galilée, Université Paris 13, CNRS, 93430 Villetaneuse,

\section{France}

(Dated: 12 July 2019)

The distribution function of electrons accelerated by intense laser pulses at steep vacuumplasma interfaces is investigated by using Fokker-Planck equation and methods from extreme statistics. The energy spectrum of electrons penetrating into the dense plasma after being accelerated at the interface and in the pre-plasma shows systematically cutoff-like decrease in the momentum component $p_{x} / m_{e} c$ along the laser propagation axis. While the distribution associated to the kinetic energy spectrum $\left(E_{k i n}\right)$ is often approximated by a thermal distribution, $F\left(E_{k i n}\right) \propto \exp \left(-E_{k i n} / T_{h}\right)$, with a hot particle temperature $T_{h}$, the nature of the distribution close to the cutoff is clearly non-thermal. Electron distributions are analyzed here from two-dimensional Particle-in-Cell simulations. Via a comparison with solutions derived from a Fokker-Planck equation and based on Chirikov's standard map models, we find that the electron distributions show a clear signature of stochastic heating, due to repeated acceleration in the standing wave in the pre-plasma. Further analysis of the solutions to the Fokker-Planck equation allows us to describe the cutoff seen in the momentum $p$ of the distributions $F(p)$, which can be expressed as a function of time $\tau$ in the form $F(p, \tau) \propto\left[\left(p_{\max }-p\right) / \delta p\right] \exp \left(-2 p^{3} / 9 \tau\right)$, portraying a time-dependent cutoff at $p \rightarrow p_{\max }$. This implies that the energetic tail of the distribution belongs to the maximum domain of attraction of the Weibull law, which means that the probability to find highenergy electrons varies abruptly near $p_{\max }$. The variance of physical observables sensitive to the high-energy tail is consequently considerably higher than when assuming thermal distribution.

a) This article has been accepted for publication in Physics of Plasmas. After it is published, it will be found at https://aip.scitation.org/journal/php 


\section{INTRODUCTION}

Energetic electrons that have been accelerated at vacuum-plasma interfaces by intense laser pulses $^{1-3}$ and which penetrate into the dense plasma are nowadays often used to accelerate ions at the rear surface of the target. The most prominent process associated with the acceleration at the vacuum-plasma interface is TNSA ('Target Normal Sheath Acceleration'), and one of the most fructuous applications is proton acceleration that has been investigated abundantly and is nowadays applied for numerous diagnostic purposes ${ }^{4-14}$.

Those electrons that enter into the laser electromagnetic field under "favorable" conditions, $\mathrm{i}$. e. entering the laser field at an optimum phase, obtain a transfer of momentum from both the laser field and the electrostatic field that form at the vacuum-plasma interface. ${ }^{15-19}$ or via the action

of counter-propagating laser fields. ${ }^{20-23}$ With the gained momentum the electrons escape from the influence of the fields that become rapidly evanescent in the skin layer. Due to this process, bunches of energetic electrons are injected into the dense medium with a period corresponding to one or $1 / 2$ of the laser field cycle ${ }^{24}$. The maximum momentum and energy that can be transferred via the laser fields and the electrostatic field to electrons is limited, as we explain later in section III. Estimating the maximum achievable energy from a purely ponderomotive picture for the acceleration processes ${ }^{1}$, that does not include longitudinal oscillations, would predict electron energies on the order of the oscillation energy of the laser field, namely $\gamma_{\text {osc }}=\mathscr{E}_{\text {osc }} /\left(m_{e} c^{2}\right) \sim$ $\left[1+I_{L} \lambda^{2} /\left(1.37 \times 10^{18} \mathrm{Wcm}^{-2} \mu \mathrm{m}^{2}\right)\right]^{1 / 2}$, with $m_{e}$ as the electron rest mass, $c$ the speed of light, $I_{L}$, $\lambda$ as the laser intensity and wavelength, respectively.

It has however been observed in simulations ${ }^{25-29}$ that the highest momentum and energy of electrons may evolve in time, while this depends on the complexity of the processes that arise in the vicinity of the interface. For "relativistic" laser fields, the number of electrons that are pushed into the relativistic energy regime increases strongly with the laser intensity 1,25,26,30-34. The probability to find electrons at relativistic energies strongly increases with the laser intensity with respect to the population of the initially thermal electron.

It has clearly been seen in simulations ${ }^{25-30,33-35}$ that distribution functions of laser-accelerated electrons show an upper bound in the particle momentum $p /\left(m_{e} c\right)$. While the nature of this upper bound is difficult to identify in Particle-in-Cell (PIC) simulations, because of the limited number of particles in the tail of the distribution, ${ }^{25-27,30,33-35}$ it is striking that Vlasov-Maxwell simulations bring to evidence non-Gaussian statistics and cutoff behavior for the electron distribution. ${ }^{28,29}$ 
From such studies it follows that the upper bound in the spectrum of the accelerated electrons scales with the laser intensity, and depends furthermore on the incidence angle, the density gradient of the vacuum plasma interface, and on the complexity of the surface structure.

We examine here the nature of the distribution function of electrons that have been accelerated inside the dense plasma, and, by means of extreme statistics, we particularly investigate the tail of this distribution. For this purpose we have analyzed results from Particle-in-Cell (PIC) simulations performed with the code $E M I 2 D$ in two spatial dimensions (2D), $x$ and $y$, and three dimensions in momentum space $(3 \mathrm{~V})$ for a case where the intensity of the laser pulse drives electrons into the regime of relativistic oscillations. In this simulation we clearly see a cutoff like behavior for the upper bound in the electron momentum.

The article is organized as follows. In section II we present our reference simulation, in section III we review models for acceleration mechanisms in the regime stochastic heating which lead to standard map models. Derived from this, in section IIIC we use a Fokker-Planck (FP) equation. We analyze a family of distributions and probability densities which are solutions to FP equations and we propose a realistic distribution function for the electron momentum which is consistent with simulations. Furthermore in III D we verify the model against standard map simulations using the parameters deduced from the PIC simulations. In section IV we investigate the limit law of the energetic tail of the momentum distribution, and in $\mathrm{V}$ we discuss the results and conclude.

\section{REFERENCE SIMULATION}

The reference simulation case discussed here corresponds to the case as discussed previously in Ref. 36. In the discussed simulation the laser intensity was $I_{L}=1.23 \times 10^{19} \mathrm{~W} / \mathrm{cm}^{2}$, at the wave length $\lambda=1 \mu \mathrm{m}$, arriving at the interface at $\omega_{0} t=140$ and reaching its peak value at $\omega_{0} t=200$, where $\omega_{0}$ stands for the laser frequency. The pulse then remains constant until the end of the simulation at $\omega_{0} t=900$, corresponding to an approximate pulse duration of $\sim 300 \mathrm{fs}$. The laser intensity corresponds to a value of the normalized vector potential $a_{0}\left[=e E_{L} /\left(m_{e} \omega_{0} c\right)\right] \simeq 3$ with $E_{L}$ as the peak amplitude of the oscillating laser field in vacuum, polarized in $y$-direction $(p$ polarisation, normal incidence). The plasma behind the interface, $k_{0} x>150$, with $k_{0} \equiv 2 \pi / \lambda$, has an electron density of $n / n_{c}=100$ times the critical density, $n_{c}$, with a sharp gradient of $(2 \lambda)^{-1}$.

An underdense pre-plasma forms gradually left of the interface initially situated at $k_{0} x \simeq 136$. For the chosen case a major part of the laser light is reflected after a transient period. In the PIC 

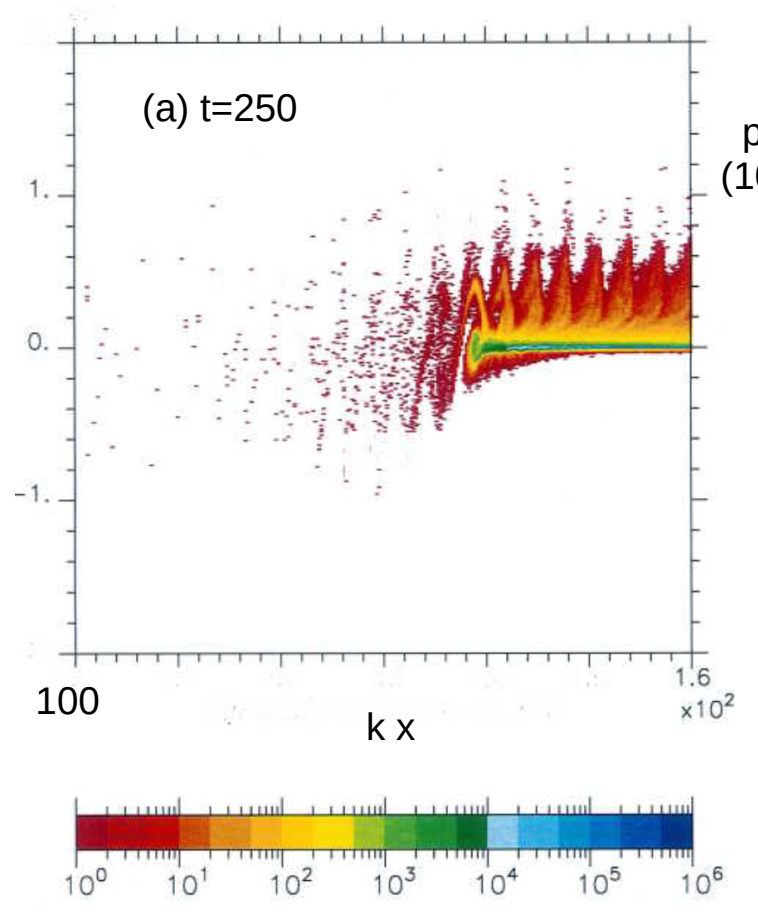
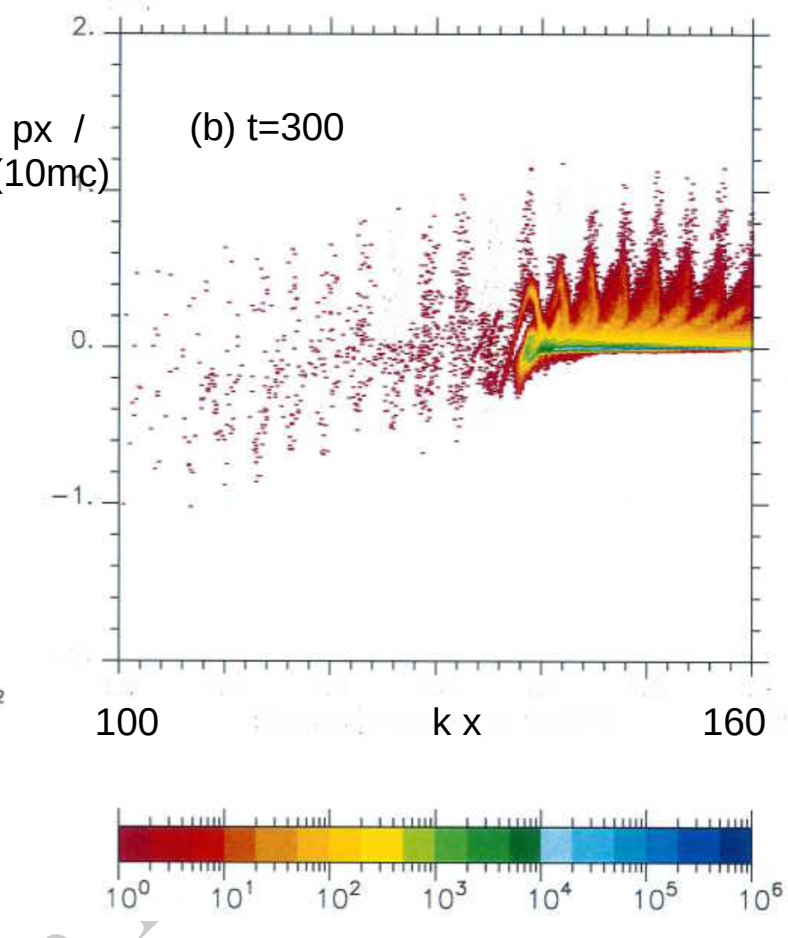

FIG. 1. Phase space snapshot of $2 \mathrm{D}(3 \mathrm{~V})$ PIC simulations taken at (a) $\omega t=250$ and 300 , along the laser propagation axis in $x$ and $p_{x}$ for $y=y_{0}$ (on axis in the second spatial dimension) in the vicinity of the vacuum-plasma interface.

simulation, light is reflected at the interface with a reflection coefficient of $r_{\mathrm{PIC}} \simeq 0.83$ in amplitude (i.e. $r_{\text {PIC }}^{2} \simeq 70 \%$ in light flux) after $\omega_{0} t \simeq 200$. The reflection coefficient stays almost constant for the rest of the simulation. This forms hence an important reflected light component and so an almost standing electromagnetic field in the pre-plasma. Figure 1 shows the phase space of electrons, in $k_{0} x$ and $p_{x} /\left(m_{e} c\right)$ for the time instant of $\omega_{0} t=300$. For the analysis of simulation data we focus only on electrons close to the center of the laser spot, so that the part of the electrons shown from this two-dimensional (2D) simulation are found in close vicinity to the axis where the laser pulse impinges on the target. Beyond the shelf-solid interface at $k_{0} x \simeq 136$ electrons essentially have positive momenta $p_{x}>0$ and penetrate into the dense plasma. This is further analyzed later and shown in more detail with the help of distribution functions.Left of the interface electrons show phase-space bursts with both negative and positive values in the momentum. Electrons have been ejected from the interface into the negative $x$-direction and propagate now away from the interface. ${ }^{27}$ At the high energies achieved, already in a relativistic regime, they propagate almost with light speed towards the vacuum, but undergo influence both of the counter-propagating light 


\section{$130<\mathrm{k}_{0} \mathrm{x}<136$}

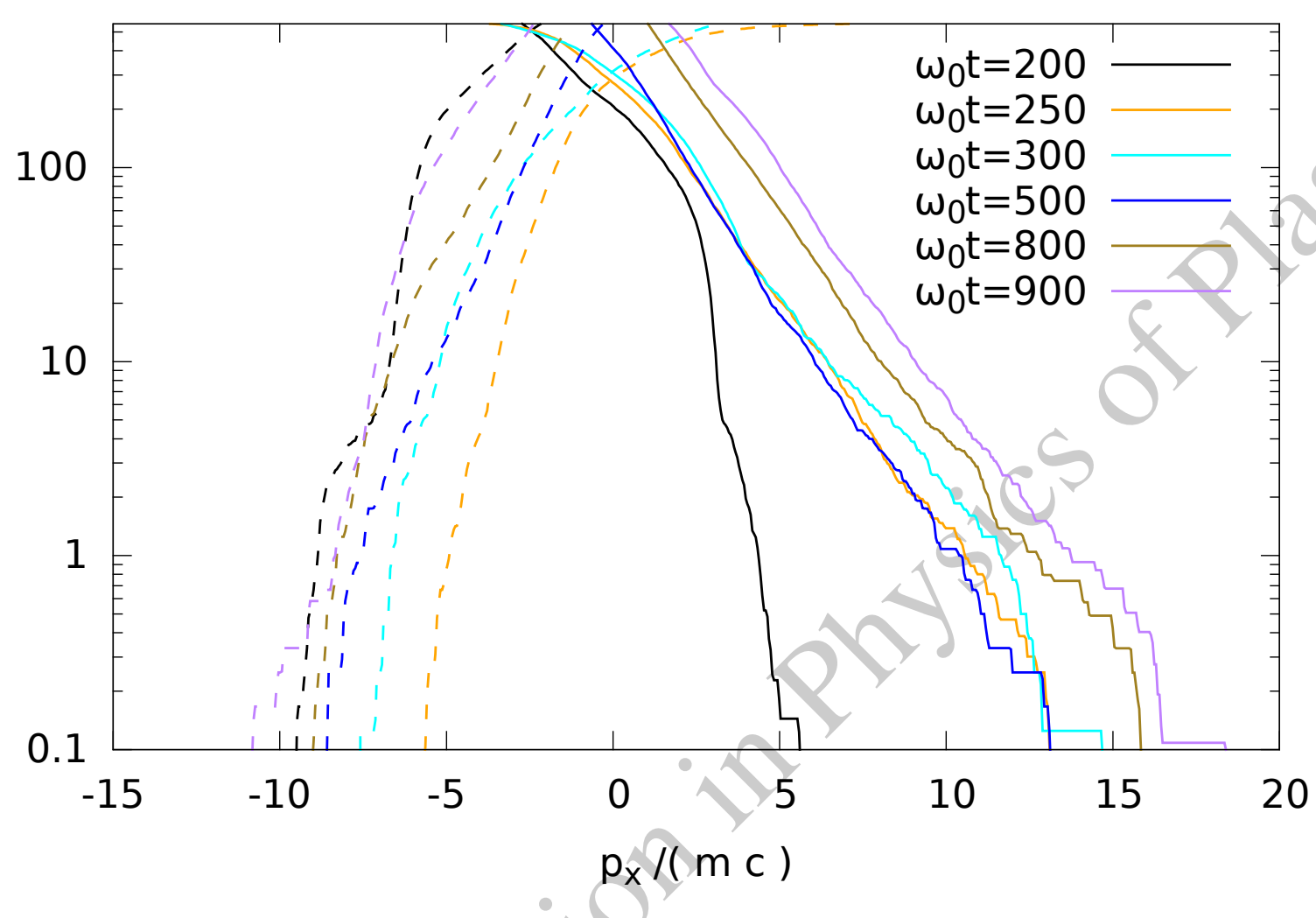

FIG. 2. Electron distribution functions $\bar{F}_{+}\left(p_{x}\right)$ (solid lines) and $\bar{F}_{-}\left(p_{x}\right)$ (dashed lines) as defined in the text, and multiplied with the total number of particles counted $(\sim 1000)$ in the considered phase-space interval in $130<k x<136$ for time instants $\omega_{0} t=200$ (black), 250 (orange), 300 (light blue), 500 (dark blue), 800 (olive), and 900 (purple). Times $\omega_{0} t=250$ and 300 correspond to Fig. 1.

fields and the ambipolar field that forms gradually due to the charge separation from the ions. Besides the oscillatory force in $y$ of the light field, and the ambipolar field along the $x$-direction, a ponderomotive force with a periodicity of $\lambda / 2$ along $x$-direction is established due to the reflected light for $\omega_{0} t>140$. From Figure 1 one can observe electron bursts in which the momenta increase 7-9 times when propagating away from the interface. At a certain distance from the interface, very few electrons with $p_{x}<0$ are found to still propagate into the negative $x$-direction close to laser axis, i. e. inside the focal spot of the incoming laser. A major part of the electrons now propagate with positive momenta $p_{x}>0$ toward the interface. In Figure 2 we show the electron distributions at different time instants, determined from the phase space in the interval $130<k_{0} x<136$ 
showing both the part of electron propagating away from the shelf-solid interface towards the vacuum, and the electrons entering into the dense plasma. For each population, $p_{x}<0$ and $p_{x}>0$, we have established the distribution functions from the density $f\left(x, p_{x}\right)$ in the phase space, namely $\bar{F}_{-}\left(p_{x}\right) \equiv \int_{p_{x}=-20}^{20} d p_{x}^{\prime} \int_{130<k_{0} x<136} f\left(x^{\prime}, p_{x}^{\prime}\right) d x^{\prime}$ and $\bar{F}_{+}\left(p_{x}\right) \equiv \int_{p_{x}=20}^{-20} d p_{x}^{\prime} \int_{130<k_{0} x<136} d x^{\prime} f\left(x^{\prime}, p_{x}^{\prime}\right)$, respectively; i.e. they show the increase in the electron distribution both in the positive and the negative branch of $p_{x} / m_{e} c$. The curves of $\bar{F}_{+}\left(p_{x}\right)$ and $\bar{F}_{-}\left(p_{x}\right)$ intersect at the instantaneous oscillation center of the "cold" electrons. For times $\omega_{0} t>200$, the cutoff values in the positive branch $\bar{F}_{+}\left(p_{x}\right)$ close to the interface are systematically higher than the cutoff values in $\left|p_{x}\right|$ in the negative branch $\bar{F}_{-}\left(p_{x}\right)$.

Figure 3 illustrates structures of electron bursts periodically injected into the dense plasma. The colors indicate the contour values which correspond to the probability density $p d f, f\left(p_{x}, x, t\right)$ (in a.u.) in $x$ and $p_{x}$. The colors are saturated for the cold electron population in order to emphasize the density of the energetic electrons. The red line in the positive $p_{x}$-half space corresponds to the momenta $p_{\max }(x)$ associated to the fastest electrons at the respective position in $x$. Beyond this line no electrons were counted in the $p d f$ determined from the PIC simulation for $p>p_{\max }$ at the respective position, i.e. $f\left(p_{x}>p_{\max }(x), x, t\right)=0$. The red crosses found at the bursts in the phase space indicate the peak momentum $p_{\max }\left(x_{n}\right)$ in the spatial interval, $n \pi / 2 \leq k_{0}\left(x_{n}-x_{0}\right)<$ $(n+1) \pi / 2$, corresponding to the periodic injection of electrons in the dense plasma, pronounced in a spatial periodicity $\approx 2 \pi c / \omega_{0}$, with $x_{0}=150 / k_{0}$, and with $n$ as the index of the successive bursts. We will see later (see section IV) that the histogram of these peak values constitutes the extreme statistics corresponding to the motion of energetic electrons penetrating into the dense plasma. We have determined the distribution of electrons as a function of momentum $p_{x}$ and space $x$ at different time instants. For the phase space plots, Figs. 1 and 3 and the particle statistics shown in Figs. 2 and 4 we integrated or averaged, respectively, over $N_{y}=14$ data sets from grid points in the direction $y$ transverse to the incident laser, within the interval $-30 \lesssim k_{0}\left(y-y_{0}\right) \lesssim 30$, corresponding approximately to the focal spot around the laser axis at $y_{0}$.

The data sets of Fig. 2 and Fig. 4 were taken within the $x$-interval close to the vacuumplasma interface, $130<k_{0} x \leq 136$ and $150 \leq k_{0} x \leq 250$, i.e. in front and behind the interface, respectively. In the interval $150 \leq k_{0} x \leq 250$ the peak values of $p_{x}$ observed in the electron bursts do not considerably decrease. The choice of the interval guarantees hence for sufficiently good statistics. ${ }^{37}$.

In order to focus on the relevant data of the probability density which can clearly describe the 


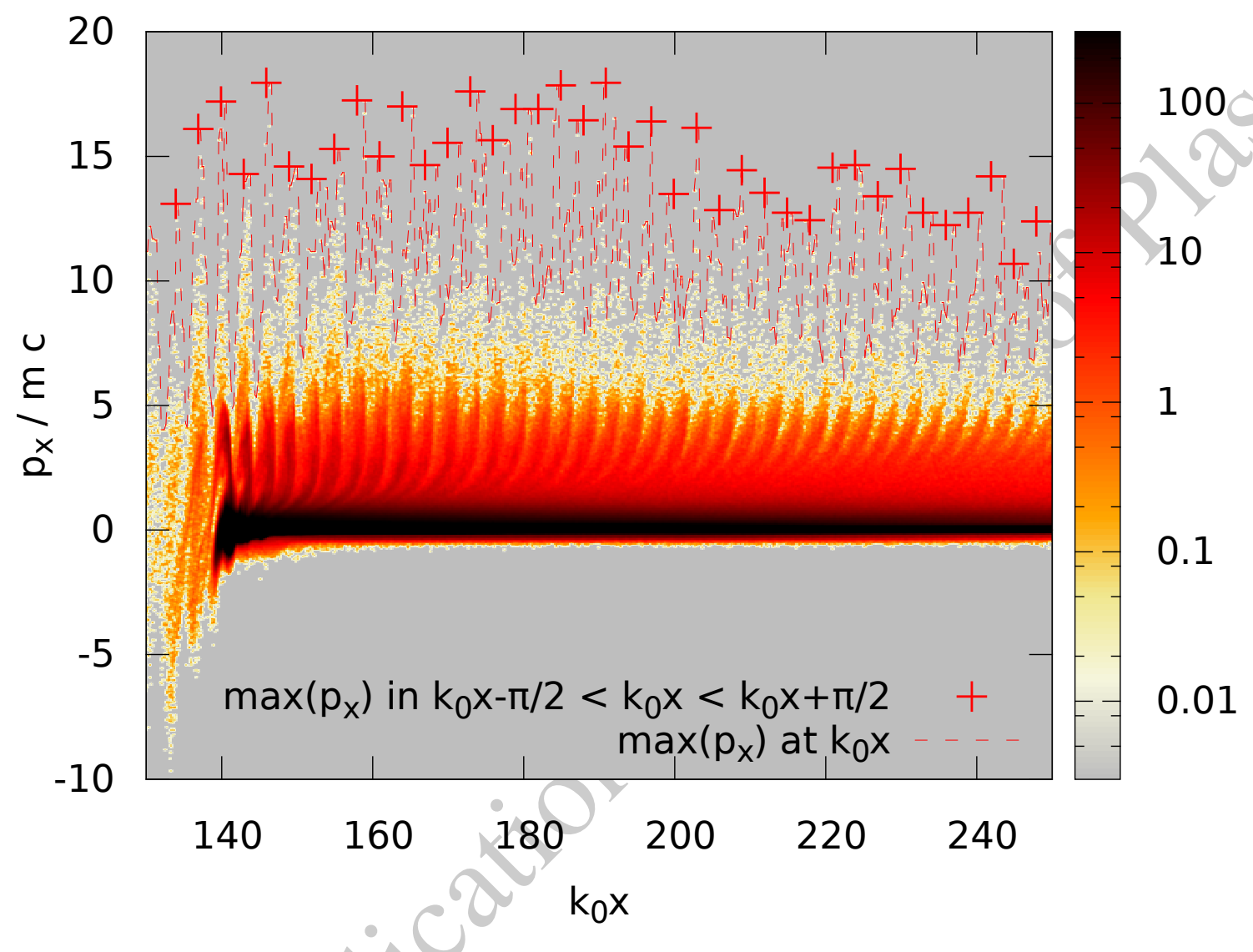

FIG. 3. Phase space snapshot of $2 \mathrm{D}(3 \mathrm{~V})$ PIC simulations taken at $\omega_{0} t=600$, along the laser propagation axis in $x$ and $p_{x}$ for $y=0$ (on axis in the second spatial dimension). The red dashed line indicates the highest electron momentum at the respective position in $x$; the values marked with ' + ' indicate the highest momentum observed in $\lambda / 2$ intervals.

behavior in the tail of the distribution, we retain two types of data sets, namely

(i) the $p d f$ values $f\left(p_{x}, x_{n}, t\right)$ at positions $x_{n}$ where a burst of electrons appears, i. e. as mentioned earlier, in about $N_{x}=36$ intervals of $x_{n+1}-x_{n}=\pi / k_{0}$ within $150<k_{0} x_{i}<250$ and

(ii) the histogram of the values $p_{\max }\left(x_{i}\right)$-values taken at all $x$-positions $x_{i}\left(=x_{i-1}+\Delta x\right.$, with $\left.\Delta x=0.2 / k_{0}\right)$ of the phase space in the interval $150<k_{0} x_{i}<250$. This yields the complementary distribution of the maxima in $p_{x}$ at each $x_{i}$, given by $\bar{F}_{h}\left(p_{\max , \mathrm{i}}, t\right)=\sum_{i=1}^{\mathscr{N}} f\left(p_{\max }\left(x_{i}, t\right)\right) / \mathscr{N}$, 
with $\mathscr{N}=N_{y} \mathscr{N}_{x}$ and $\mathscr{N}_{x}=500=(250-150) /\left(k_{0} \Delta x\right) \gg N_{x}$.

We have extracted from the phase space the $p d f$ data at different time instants. For each time, the complementary distribution functions $c d f$ from the set (i) is defined as $\bar{F}\left(p_{x}, t\right) \equiv$ $\int_{p}^{\max \left(p_{\max }\left(x_{n}\right)\right)} \sum_{n \leq N_{y} N_{x}} f\left(p_{x}^{\prime}, x_{n}, t\right) d p^{\prime}$ for $p_{x}>0$, where $\max \left(p_{\max }\left(x_{n}\right)\right)$ denotes the highest momentum value observed in all bursts and where $N_{x}$ indicates the number of bursts seen along the chosen $x$ interval. Bursts appear with an approximate periodicity of $\pi c / \omega_{0}$, yielding $N_{x}=36$ independent data sets within $150 \leq k_{0} x \leq 250$. In Figure 4 we show the complementary distribution functions $c d f$ from the set (i). The computation of the $c d f \bar{F}\left(p_{x}, t\right)$ is hence based on $N_{y} N_{x}=14 \times 36$ independent data subsets for the time instants $\omega_{0} t=200$ (shortly after the full onset of the laser pulse), 300, 400, 500 and 600. In the same plot we show, alternatively, the $c d f$ according to set (ii), namely, $\bar{F}_{h}\left(p_{x}, t\right)$ as mentioned above determined from the values $p_{\max }\left(x_{i}\right)$ of all $x_{i}$ positions of the $N_{y}=14$ data sets. Note that during the simulation particles with $p_{x} /\left(m_{e} c\right)>20$ were not stored; therefore also the distribution functions are unknown for $p_{x} /\left(m_{e} c\right)>20$. However, electrons with $p_{x} /\left(m_{e} c\right)>20$ do not occur in the simulation before times $\omega_{0} t \sim 500$ for the current case. The comparison between both sets of curves, $\bar{F}$ and $\bar{F}_{h}$, shows that the $c d f, \bar{F}_{h}\left(p_{x}, t\right)$ determined from the $f\left(p_{\max }(x)\right)$-values covers clearly the energetic and relativistic tail of $\bar{F}(p, t)$, in particular for $p>3 m_{e} c$. Figure 4 shows that the distribution evolves in time. We pay particular attention to the high-energy tail, in which one can observe a limit value in the momentum $p_{x}$ that increases in time $p_{\max }(t)$.

At each time instant the distributions show a cutoff like behavior in $p_{x} /\left(m_{e} c\right)$, for which we depict the value $p_{\max }$ in Figure 5 as a function of time. The origin of the cutoff seen in the data could a priori be two-fold : (a) a 'real' cutoff due to a maximum momentum associated with a limit value beyond which electrons cannot be accelerated, and/or (b) a cutoff due the numerical resolution, i.e. due to limited number of particles in the PIC simulation, and associated to the lowest contour value available. A cutoff of type (a) would correspond to a distribution of energetic electrons not following Gaussian statistics. Figure 5 illustrates that the value of the observed cutoff, i.e. $p_{\max }$, increases with time. To underline this we have superposed to the data a curve that follows a power law in time with $\propto t^{2 / 3}$. However, with respect to this power law, the increase of $p_{\max }(t)$ in time clearly slows down for $\omega_{0} t>450$. These power-law behavior, whose exponent value $2 / 3$ will be motivated later on in section III C 2, fits very well the observed time evolution of the cutoff up to $\omega_{0} t \simeq 450$. Figure 5 also shows the expectation value in $p_{x}$ of the energetic electrons in $\bar{F}_{h}$, which will be discussed in section III C 2 . 


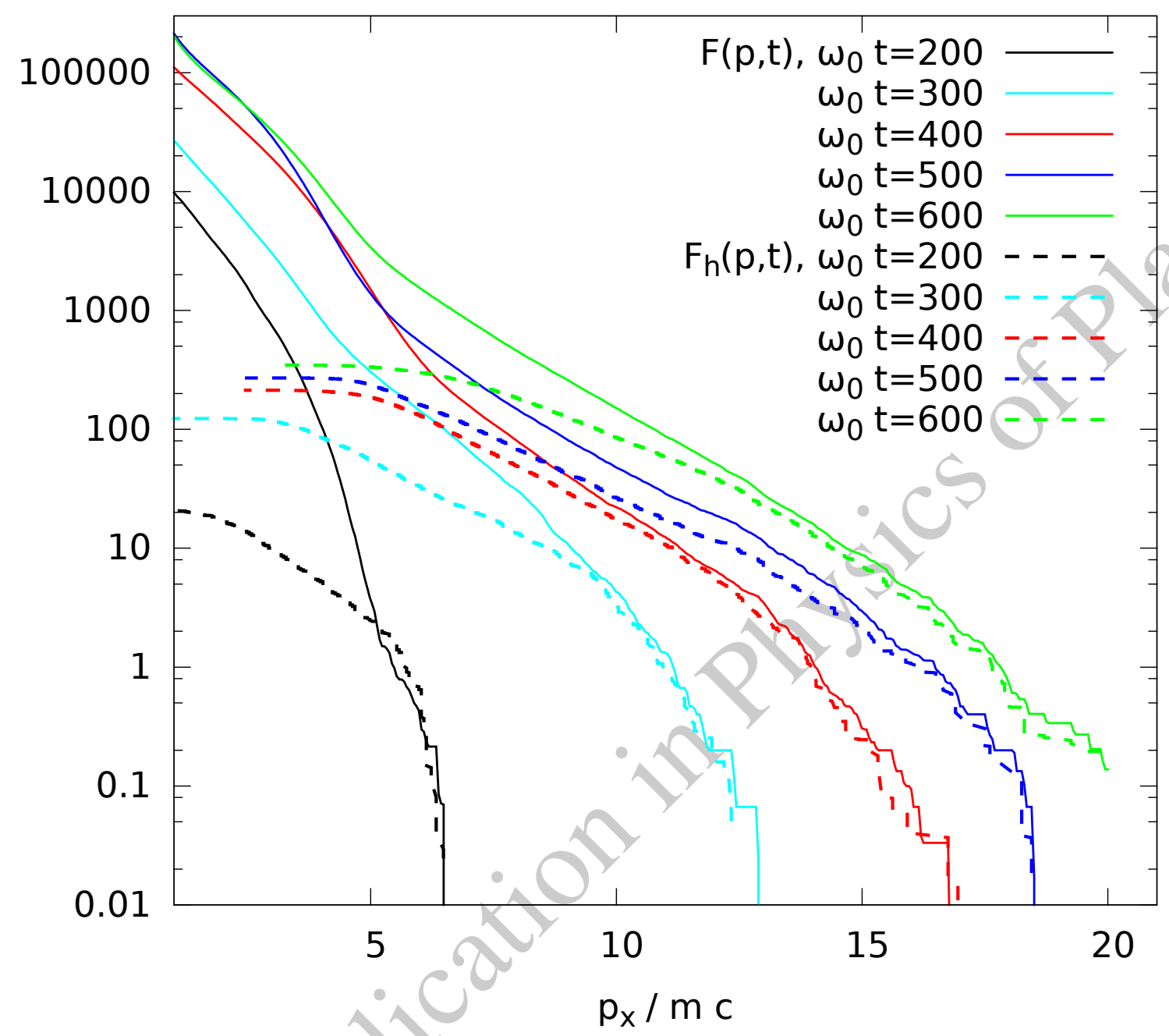

FIG. 4. Complementary distribution functions $\bar{F}(p, t)$ (solid lines) and $\bar{F}_{h}\left(p_{x}, t\right)$ (dashed lines), multiplied with the total number of particles counted in the considered interval of the PIC simulations, for different time instants, $\omega_{0} t=200,300,400$, and 500. The values corresponding to $\bar{F}_{h}\left(p_{x}, t\right)$ are data sets obtained on the basis of the points in $x$ of the red curve in Fig. 3 delimiting the highest $p$-value seen in the phase space.

With the help of the distributions shown in Fig. 4 given at 5 different time instants, between $\omega_{0} t=200$ and 600, we have determined the values of $p=p_{c, i}$ corresponding to 6 different isocontour levels $F\left(p_{c, i}, t\right)=c_{i}$ in between $c_{1}=0.2$ and $c_{6}=100$ well above the observable cutoff level $c_{\text {cutoff }}<c_{i}$. The evolution of the corresponding momenta $p_{c, i}(t)$ in time proves to be slower than seen for the cutoff $p_{\max } \propto t^{2 / 3}$.

Refering to models for diffusion in momentum space ${ }^{38,39}$, the evolution of the distributions 


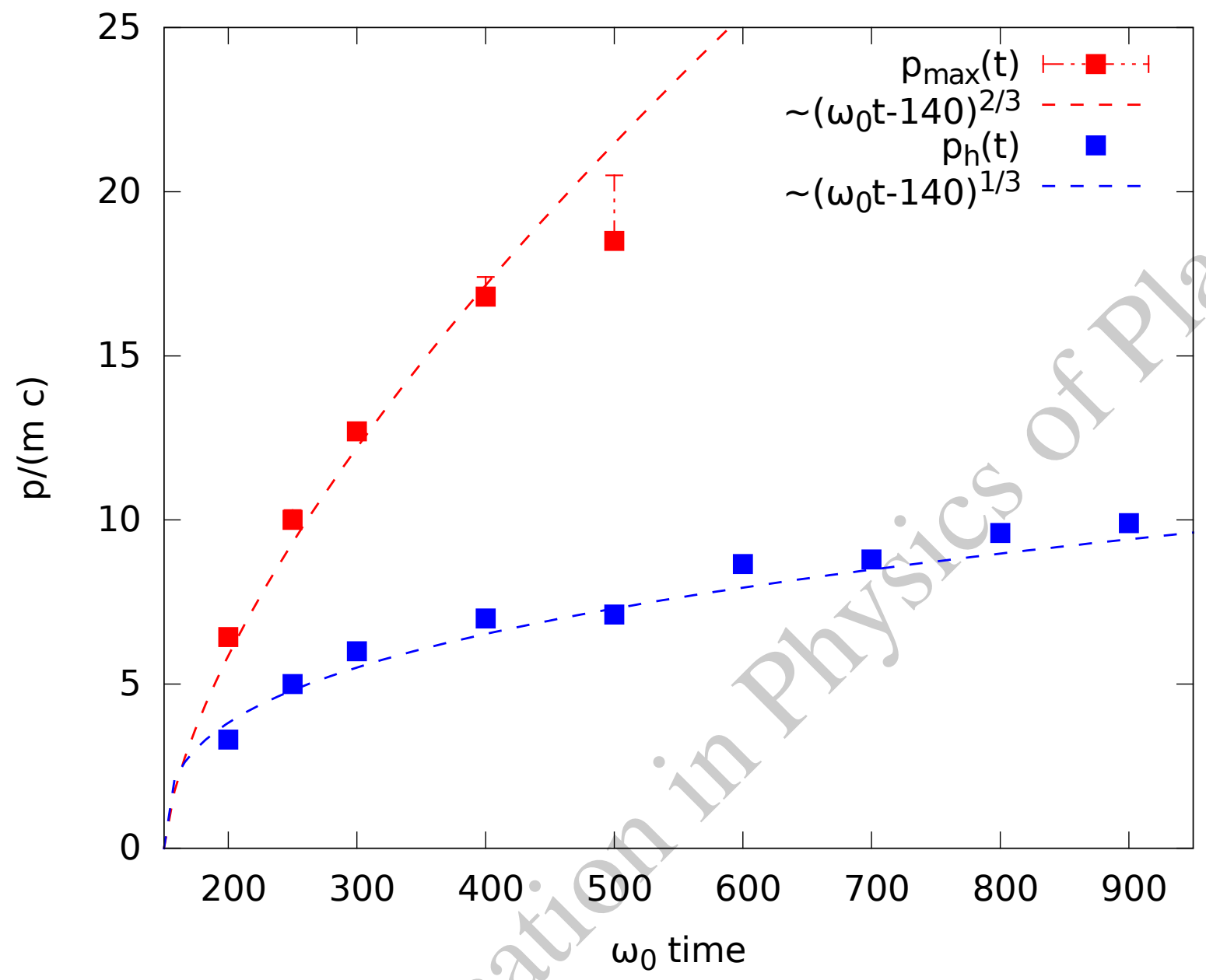

FIG. 5. Values of the highest observable electron momentum $p_{\max }$ (red points) from the distributions $\bar{F}\left(p_{x}, t\right)$ and $\bar{F}_{h}\left(p_{x}, t\right)$ in Fig. 4 as well as the expectation values in momentum, $\bar{p}_{h}$ (blue points), of the energetic electrons corresponding to $\bar{F}_{h}\left(p_{x}, t\right)$ from PIC simulations in the interval behind the vacuumplasma interface, for different time instants. The red dashed line shows, as a guide line, the evolution of the contour for a cutoff behavior following a power law behavior $\propto t^{2 / 3}$, the blue dashed line to the power law evolution $\propto t^{1 / 3}$. Note that for advanced times, $\omega_{0} t>500$, the contour where a cutoff was observable is below the resolution of the PIC code.

follows a diffusion-like behavior, $\bar{F}(p, t) \propto \exp \left\{-p^{2} / D t\right\}$ we have numerically determined from the iso-contour values the diffusion coefficient $D$ belonging to this type of solutions. In $\bar{F}(p, t)$ we neglect for simplicity power-law dependence on $p$ and $t$. The inverse of the diffusion coefficient for each data point of the $i=1 \ldots 6$ contour values and 6 time instants is then determined by computing $D^{-1}=-\left(t / p_{c, i}^{2}\right) \log \left[F\left(p_{c, i}, t\right)\right]$. The resulting values of the diffusion coefficient from the data 


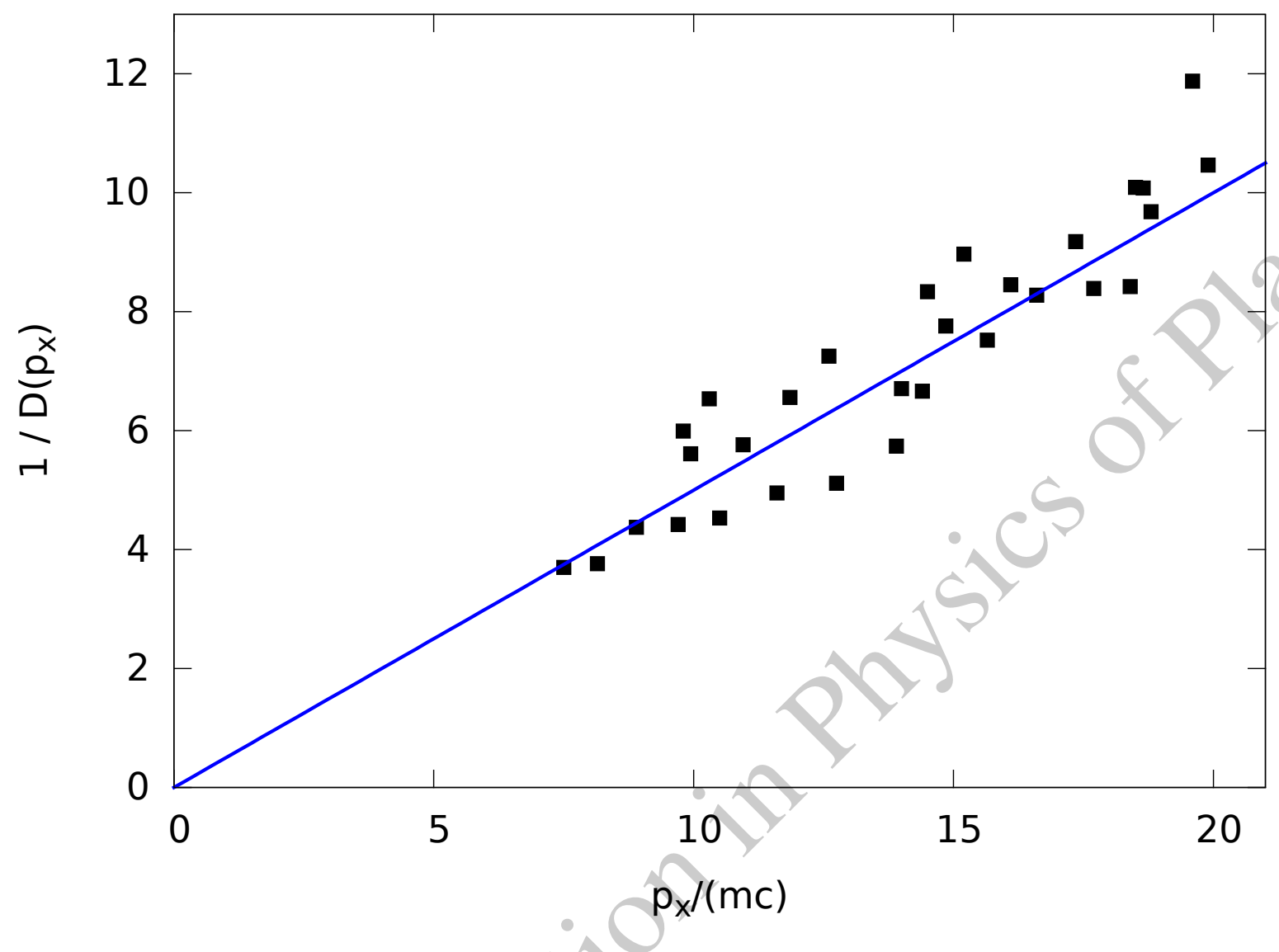

FIG. 6. Inverse of the diffusion coefficient $D^{-1}$ associated with the exponential behavior $\propto \exp \left(-p^{2} / D t\right)$ for the distribution $\bar{F}(p, t)$, deduced from iso-contour values of Fig. 4 at different time instants. As a guideline, a linear increase with $\propto p$ for $D^{-1}$ is drawn.

extracted of Fig.4 are drawn in in Fig. 6, and they show a clear dependence of $D^{-1}$ on $p_{x}$, i. e. following a linear increase in $p_{x}$ for the interval of interest $5<p_{x} /\left(m_{e} c\right)<20$. This means that the energetic tail of the distribution should be dominated by the exponential dependence $\sim$ $\exp \left\{-p^{2} / D(p) t\right\}=\exp \left\{-p^{3} / \kappa t\right\}$, with $D(p) \equiv \kappa / p$, except for the high-energy tail close to the cutoff $p \rightarrow p_{\max }(t)$ in which the decrease is faster. We discuss this in more detail in section III C 2 .

Our goal is to understand the nature of the energetic tail and the limit law in terms of extreme statistics ${ }^{40}$, which unequivocally allows us to distinguish the distribution associated with thermallike Gaussian statistics, and non-thermal, non-Gaussian statistics. For this purpose we discuss in the following the possible acceleration mechanisms involved. The acceleration of the electrons up to highly relativistic momenta takes place both at the interface between the solid-density plasma 
and the pre-plasma as well as in the pre-plasma where waves still propagate. Electrons that are eventually accelerated and injected into the dense plasma are hence exposed to the ponderomotive force in counter-propagating light fields, to the electrostatic field from charge separation between electrons and ions, and to the effective electric driver field in the skin layer. It should be mentioned that kinetic heating mechanisms have been identified inside the dense plasma behind the skin layer ${ }^{36,41}$, where the high energy electron bunches coming from the interface excite a wake fieldtype plasma wave, which in turn provokes wave-particle interaction.

An important aspect for the understanding of the motion of energetic electrons in this article will be the potential mechanisms for stochastic acceleration. In the context of superposed light fields numerous, mostly theoretical studies have been undertaken ${ }^{20,22,23,42-44}$, several particularly focused on the situation considering incident and reflected light waves having the same frequency. ${ }^{21,23,27}$ The formation of electron bursts originating from electron acceleration in the vicinity of the interface has been widely discussed. ${ }^{18,23,24,26,45-48}$ Essentially, the description of the electron motion at the interface between pre- and solid-density plasma can be condensed into a model that considers the motion in both an oscillating electric driver field ${ }^{18,45}$, and a self-generated inhomogeneous electric field. ${ }^{15,16,18,46,49}$ In Ref. 50 this type of motion has been discussed and elaborated, resulting in a description similar to Chirikov's standard map and to diffusion in momentum space. In the work by Bulanov et al. ${ }^{38}$ a simplified description by conserving the essential physics is elaborated, with further analysis of the phase space diffusion. The models for evoking stochastic heating mechanisms, both the acceleration in superposed light waves and the acceleration combining a driver field and an electrostatic potential, can be reduced to an essentially one-dimensional picture. Two-dimensional (2D) features, definitely present in the 2D (3V in velocity space) simulations, have to be taken into account in the context, in particular what concerns the number of particles that circulated in the vicinity of the interface.

To take into account all the different acceleration mechanism described above, we discuss in the following models that were developed in the context of both the acceleration in the layer of the interface and the acceleration in the pre-plasma in presence of superposed counterpropagating light waves. 


\section{MODEL AND STOCHASTIC HEATING}

While the motion of electrons under the influence of an intense laser pulse at the pre- to soliddensity plasma interface can be quite complex, several attempts have been made to reduce this motion to the essential effects. Bulanov et al. (2015) and Paradkar et al. (2012) $38,50,51$ have developed models that explain the formation of energetic tails in the electron distribution function based on stochastic acceleration processes. Their models combine the periodic acceleration in the laser field with the motion in the electric field due to charge separation at the sharp plasma boundary. The equation of motion for electrons in $x$-direction can then be presented in the form

$$
\frac{d p_{x}}{d t}=-\frac{1}{2 \gamma} \frac{\partial\left(a_{\perp}\right)^{2}}{\partial x}-e E_{x},
$$

where $a_{\perp}$ is the electromagnetic vector potential, $E_{x}$ the electrostatic field due to charge separation, and the relativistic factor being defined as $\gamma^{2}=1+\left(p_{x}^{2}+\left\langle\left(p_{y}+a_{\perp}\right)^{2}\right\rangle\right)$ with time averaging \langle\rangle .

From our reference simulation it can be seen that the initial acceleration of electrons starts from the interface with a steep density gradient. After initial acceleration in the skin layer, electrons can propagate both into the direction of the dense plasma as well as into the direction of the vacuum. A part of the electrons immediately propagate into the dense plasma with sufficiently high energy such that they escape from the influence of the electromagnetic light field. The electrons propagating towards the vacuum, however, remain under the influence of the incident and reflected light waves. The effect of the ponderomotive force on the density gradient has eventually an important influence on the number of electrons that are stochastically accelerated in the standing wave. The effects in one- or two (three) dimensional geometry have clearly to be distinguished (see also 30): in 1D, the ponderomotive force due to the field amplitude variation in the skin layer, $\propto \nabla\left|a_{\perp}\right|^{2}$, provokes important profile steepening in the $x$-direction on both the electron and the ion density profile, such that particles cannot escape from this force. As a result, few electrons escape towards the vacuum region. In 2D (or 3D), hole boring or filamentation effects will arise by locally increasing the laser intensity, such that profile modifications are not homogeneous in $y$ along the target surface. A non-negligible fraction of electrons with non-zero values in $p_{y}$ may escape from regions where the ponderomotive force in $x$ is strong, which are then laterally injected into the vacuum region, eventually getting under the influence of the standing wave ${ }^{30}$.

In the following we consider the electron motion in the electromagnetic fields and distinguish their motion in the vicinity of the interface between the very low-density pre-plasma and the dense 
plasma layer, which we index as case ' $i$ ", and the motion in the pre-plasma dominated by the superposed fields of the incoming and reflected light, indexed as case ' $t w$ '. The associated models to these cases are essentially one-dimensional.

\section{Interface case ' $i$ ',}

In Ref. 38 a model Hamiltonian has been derived in a condensed manner for 1D relativistic electron motion in an electrostatic potential $U(x)$ in the presence of an oscillating driver field $A_{d}(x)$ that is associated to an electromagnetic wave:

$$
H\left(x, p_{x}, t\right)=\sqrt{1+p_{x}^{2}}+U(x)-A_{d}(x) \cos (\omega t),
$$

where $p_{x}$ is the electron momentum (in units of $m_{e} c$ ), $x$ its spatial coordinate, and where the driver field oscillates in time with the frequency $\omega$ at the amplitude $A_{d}$ that may itself depend on $x$. The aspect of acceleration for case ' $i$ ' in the frame of the model Eq. (2) has been extensively studied in Refs. 38, 50, and 51. The spatial shape of the potential depends on the plasma interface; for sufficiently thin foils one would adopt a V-shaped potential with $U(x)=\varepsilon_{p}\left|x-x_{c}\right|$, while for an extendend dense plasma with the interface situated at $x=x_{c}$ it should read $U(x)=\varepsilon_{p}\left(x_{c}-x\right)$ for $x \leq x_{c}$ and $=0$ for $x>x_{c}$, with $\varepsilon_{p}$ being the electrostatic field, considered to be constant. The driver field is taken via a dipole model as $A_{d}=a_{d} x$. The driven motion along the direction normal to the surface is well-known from resonance absorption at oblique laser incidence, for which particle acceleration has been seen ${ }^{52}$ even at non-relativistic intensities. While the driver field model has later been revisited, even for higher intensities ${ }^{47-49}$, it has been shown that a field pointing along the normal can be justified as well for normal incidence at high laser intensity. ${ }^{13,45,53}$ Later, in section III C 2, we discuss the relation between the driver field amplitude $a_{d}$ and the electromagnetic field strength $a_{0}$.

Both Paradkar et al. ${ }^{50,51}$ and Bulanov et al. ${ }^{38}$ suggest that the electron motion under the combined influence of driver field and potential can lead to repeated acceleration. Stochastic acceleration via this motion can then be described by recurrence relations via Chirikov's standard (or return) map model, in which the quotient of the parameters $a_{d}$ and $\varepsilon_{p}$ eventually determines the transition to stochasticity. The validity range of this model is however limited by both the electrostatic potential and the spatial range of the assumed driver field, namely the skin layer. Electrons that are rapidly accelerated into the positive $x$-direction to sufficiently high $p_{x}$ values, will be lost from the influence of both field components once being beyond the skin layer. Electrons ejected 
into the pre-plasma will still be under the influence of the electrostatic field, but will be rapidly out of influence of the driver field.

Stochastic and repeated acceleration at the interface should in particular be excluded for the case of ultra-short laser pulses. For short pulses it has been shown ${ }^{18,54}$ that acceleration at the interface can attain values of $p_{\max }=a_{0}^{2} / 2$, which for the ultra-relativistic case, $a_{0}^{2} \gg 1$, can be far above the values expected from the maximum acceleration, $p_{\max } \sim \gamma_{\text {osc }}$, associated with the oscillation energy ${ }^{1} \gamma_{\mathrm{osc}} \equiv \mathscr{E}_{\mathrm{osc}} /\left(m_{e} c^{2}\right)$.

\section{Two-waves case ' $t w$ '}

In the reference simulation case, as already mentioned, a non-negligible part of the incoming light field is reflected, with the reflection coefficient $r \simeq 0.83$ in the field amplitude (and $r^{2}=0.7$ in intensity). The ponderomotive force from the standing wave dominates the electrostatic field already in a wavelength-distance from the interface.

To obtain the superposition of the incident and the reflected light waves, eventually resulting in a standing wave in front of the interface ${ }^{27,30}$, requires that the laser pulse, as in our simulation, is sufficiently long lasting, in our case $\omega_{0} t>400$. The latter may not be the case for very short pulses, as e.g. in the study of Ref. 54.

As observed in the reference simulation, the motion of energetic electrons escaping from the interface towards the negative $x$-direction is therefore governed by the superposed light fields. The electron motion in the standing wave can lead to repeated acceleration and repeated change in the sign of the particle momentum $p_{x}$. Electrons, initially ejected from the interface in negative $x$-direction, can therefore return in positive $x$-direction and penetrate into the dense plasma with a net energy gain due to the stochastic acceleration.

The electron motion under the influence of two light wave fields, $A_{1}$ and $A_{2}$ is described by Eq.(1) with $a_{\perp}=A_{1}+A_{2}=a_{1} \cos \left(k_{0} x-\omega_{0} t\right)+a_{2} \cos \left(-k_{0} x-\omega_{0}^{\prime} t+\Psi\right)$ with $a_{2}=r a_{1}$ at $\mid \omega_{0}-$ $\omega_{0}^{\prime} \mid \ll \omega_{0}$ for the electromagnetic field amplitudes and frequencies, respectively. The coefficient $r$ is then the reflection coefficient of the fundamental component of the reflected light wave. Let us remark here that higher odd harmonics (3rd, 5th, etc.) in the reflected light field may be excited, as in our simulation, so that the reflection coefficient for the fundamental component is weaker as the value indicated earlier.

This type of dynamics - and the stochastic heating associated with it - has been subject of numerous studies in a similar context with two or more wave components, see Refs. 20, 22, 23, 39, 
42, 55-58, with particular attention to counter-propagating waves in Refs. 23 and 59. From these approaches, it has been pointed out that for electromagnetic wave interaction the corresponding Hamiltonian can be simplified for the longitudinal motion in $x$ as $^{23} H=\left[1+p_{x}^{2}+\left(p_{y 0}+A_{1}+\right.\right.$ $\left.\left.A_{2}\right)^{2}\right]^{1 / 2}$.

The motion under the influence of a standing electromagnetic wave is then governed by an equation of motion in the $x$-direction due the ponderomotive force of the counter-propagating linearly polarized light wave components. The ponderomotive force, dominated by the term $\propto A_{1} A_{2}$, yields primarily a spatial periodicity $\sim \sin 2 k_{0} x$, with the amplitude $\propto a_{1} a_{2}=r a_{1}^{2}=\left(2 r a_{\perp, 0}^{2} / 4\right)$. The equation of motion can hence be presented in a 2 nd order differential equation for the guiding center motion of an electron, namely 60

$$
\frac{d^{2} k_{0} x}{d\left(\omega_{0} t\right)^{2}}-\frac{\omega_{b}^{2}}{\gamma_{0}^{2} \omega_{0}^{2}} \sin 2 k_{0} x=0 .
$$

in which $\gamma_{0}=\left[1+p_{x}^{2}+a_{\perp, 0}^{2}\right]^{1 / 2}$ stands the relativistic factor for the electron entering with momentum $p_{x}$ into the standing wave ${ }^{61}$ and $\omega_{b}$ for the bounce frequency of trapped particle motion around phases $2 k_{0} x=\pi$. In the current context of relativistic motion in a standing wave we define

$$
\omega_{b} \equiv \omega_{0} \sqrt{r} a_{\perp, 0} \text { and } \omega_{\gamma}=\omega_{b} / \gamma_{0}
$$

This type of equation has been studied also in similar context for trapped particle motion, and particularly for the case of electrostatic waves ${ }^{59}$.

From the observations in our simulation in Fig. 1 due to strong acceleration at the interface, and related to the above mentioned driver model, Eq. (2), electrons enter with already a nonnegligible momentum $\left(p_{0, x}^{2} \gg 1\right)$ into the standing wave. This motion of fast electrons entering into a standing wave field has been subject of numerous studies and is associated with the KapitzaDirac effect ${ }^{62,63}$, resulting in Compton harmonic resonances and stochastic instabilities ${ }^{39}$.

The Hamiltonian and the motion related to the above-mentioned model, Eq. (3), using $J=$ $2 k_{0} d x /\left(d \omega_{0} t\right)=2 p_{x} / \gamma_{0}$ and $\phi=2 k_{0} x$, can be written as

$$
H(J, \phi)=J^{2} / 2+U_{0} \cos \phi
$$

with the amplitude of the potential $U_{0}=\omega_{b}^{2} /\left(\omega_{0}^{2} \gamma_{0}^{2}\right)$ for the present case. This type of Hamiltonian has been extensively studied by Chirikov ${ }^{55}$ as well as by Escande and Doveil ${ }^{43}$ for the non-relativistic limit (see also Ref.44, p. 249), in which also the role of perturbations to the potential via spatio-temporal oscillations $\propto \cos (\psi-\Omega t)$ and higher order has been considered. 


\section{A. Standard map model}

For systems described by Eq. (2) from case ' $i$ ' and by Eq. (5) from case ' $t w$ ', it is possible, after Chirikov $^{55}$, and in analogy to Fermi acceleration ${ }^{44,64,65}$, to write down difference equations for quantities representing momentum $P$ and phase $\phi$, and which are associated to Poincaré's standard (or return) map. This set of difference equations reads

$$
\begin{aligned}
& P_{n+1}=P_{n}+K_{s} \sin \left(\phi_{n}\right), \\
& \phi_{n+1}=\phi_{n}+P_{n+1},
\end{aligned}
$$

with the stochasticity parameter $K_{s}$, whose magnitude determines the onset of stochastic regions in the phase space, and the further transition to global stochasticity.

(i) For the interface case ' $i$ ', following Bulanov et al. ${ }^{38}$, one has $K_{s, i}=8 a_{d} / \varepsilon_{p}$, with the amplitude of the oscillating driver, $a_{d}$, and the electrostatic field strength $\varepsilon_{p}$ in the vicinity of the interface. Here, $P$ is defined as $P \equiv 4 p_{x} / \varepsilon_{p}$, and $\phi=2 \omega_{0} t$ as the phase.

(tw) For the case with standing waves, Eqs. (6-7) result by taking a time step related to the bounce period, $\omega_{0} \Delta t=C_{t w} \pi\left(\omega_{0} / \omega_{\gamma}\right)$, resulting in $K_{s, t w}=C_{t w}^{2} \pi^{2}$ together with $P=C_{t w} \pi\left(\omega_{0} / \omega_{\gamma}\right) J$ $=2 C_{t w} \pi\left(\omega_{0} / \omega_{b}\right) p_{x}$ and $\phi=2 k_{0} x$. The factor $C_{t w}$ depends on whether one counts a full period, $C_{t w}=2$, or a half period with a possible change of the sign in $p$ for $C_{t w}=1$, respectively.

The threshold for onset of stochastic particle trajectories and stochastic acceleration has been examined by Chirikov 55 . For the stochasticity parameter, from $K_{S} \gtrsim 1$ particle motion can enter the stochastic regime due to resonances, and for values $K_{S}>K_{c}$, with the critical value ${ }^{43,44,55,59,66}$ $K_{c}=(\pi / 2)^{2} \simeq 2.46$, the fully stochastic regime is attained.

From this estimate we can assume that electrons entering the standing wave will undergo stochastic acceleration. We shall discuss later on, see section $\mathrm{V}$, the important factors related to the influence of the plasma density gradient, and geometry on the number and energy of the ejected electrons.

In the Hamiltonian Eq. (5) we have neglected the presence of the electrostatic potential induced by the electron cloud, as in Eq. (2). It is, however, still present while its influence on the electron motion in a high-amplitude standing wave should mainly play a role of dephasing and deceleration for a trajectory away from the interface, or acceleration for a trajectory toward the interface. In our simulations we observe, that electrons are returned towards the positive $x$-direction around $k_{0} x \sim 100$, and only a very small fraction of very energetic electrons can definitely escape toward 
the vacuum $x \rightarrow 0$. Most of the electrons have already been returned before into the positive $x$-direction, after having been temporarily trapped in the standing wave ponderomotive potential.

One has to keep in mind that the spatial range of the model in case ' $i$ ' is very narrow in $x$. The model does not consider a limited spatial extent of the oscillating driver $\propto a_{d}$. For this reason, it would be difficult to to explain repeated acceleration of highly energetic electrons by trapping in the localized electrostatic potential for as steep density gradient as present at interfaces. The physics of this model is nevertheless important for the magnitude of the $p_{x}$-momentum of electrons entering the standing wave. A single acceleration event in the interface layer may hence already be important to attain energetic electrons that enter either with $p_{x}>0$ into the dense plasma, or enter with $p_{x}<0$ into the standing wave.

\section{B. Diffusion in momentum space}

The stochastic motion can be measured by the diffusion in momentum space, for which the average square deviation of the momentum change $\overline{\left(\Delta p_{x}\right)^{2}}$ has to be evaluated, which results for both cases in

$$
\overline{\left(\Delta p_{x}\right)^{2}}= \begin{cases}2 a_{d}^{2} & \text { for case ' } i \\ C_{t w}^{2} \frac{\pi^{2}}{8}\left(\omega_{b} / \omega_{0}\right)^{2} \text { for case ' } t w\end{cases}
$$

where, for case ' $t w$ ', we take $\overline{\sin ^{2}(\phi)}=1 / 2$ and for the time interval of momentum change $\Delta t=$ $C_{t w} \pi / \omega_{\gamma}=C_{t w} \pi \gamma_{0} / \omega_{b}$. For both cases $\overline{\left(\Delta p_{x}\right)^{2}}$ results to be independent of the relativistic factor $\gamma_{0}$

This average square deviation in $p_{x}$ is directly related to the diffusion coefficient $D=\overline{\left(\Delta p_{x}\right)^{2}} / t_{c}$ that enters into the Fokker-Planck equation ${ }^{39}$, with $t_{c}$ standing for the characteristic time scale for momentum transfer events. Resuming both cases, taking for case ' $i$ ', $t_{c, i}=C_{i}\left|p_{x}\right| / \varepsilon$ (with $C_{i}=4$ from Ref. 38), and for case ' $t w$ ', again $t_{c, t w}=\Delta t_{t w}=C_{t w} \pi \gamma_{0} / \omega_{b}$, the diffusion coefficient reads

$$
D=\left\{\begin{array}{l}
\left(2 / C_{i}\right) a_{d}^{2} \varepsilon_{p}\left|p_{x}\right|^{-1} \quad \text { case ' } i, \\
C_{t w} \frac{\pi}{8}\left(\omega_{b} / \omega_{0}\right)^{3} \gamma_{0}^{-1} \quad \text { case 'tw' }
\end{array}\right.
$$

We notice that for both cases, the coefficients are inversely proportional to $\left|p_{x}\right|$, namely $\gamma_{0}=$ $\gamma_{a}\left[1+p_{x}^{2} / \gamma_{a}^{2}\right]^{1 / 2} \simeq\left|p_{x}\right|$ for $p_{x}^{2} \gg \gamma_{a}$ with $\gamma_{a}=\left[1+a_{\perp, 0}^{2}\right]^{1 / 2}$. This typical dependence of the diffusion coefficient $D$ on $\left|p_{x}\right|$ corresponds hence to the behavior seen in Fig. 6 where values have 
been deduced numerically from the simulation. On this basis we develop in the following section solution to the Fokker-Planck equation.

\section{Fokker-Planck model}

We will now describe the electron distribution in terms of a Fokker-Planck equation via the link with the diffusion coefficient $D=\overline{\left(\Delta p_{x}\right)^{2}} / t_{c}$. We use in this paragraph for simplicity $p$ for $p_{x}$.

Based on the knowledge of the diffusion coefficient of the model, one can now derive distribution functions of the accelerated energetic electron. The Fokker-Planck equation written in the form

$$
\frac{\partial f}{\partial t}=\frac{1}{2} \frac{\partial}{\partial p}\left(D \frac{\partial}{\partial p}\right) f=\frac{B}{2} \frac{\partial f}{\partial p}+\frac{D}{2} \frac{\partial^{2} f}{\partial p^{2}}
$$

expresses the probability density $(p d f)$ of the distribution function $f$. In the case that the diffusion coefficient depends on $p$, also the term with the coefficient $B$ in Eq. (10) has to be considered because of $B=\partial D / \partial p$. We concentrate in the current study to electron momenta $p=p_{x}>0$ in the positive half space of the phase space (due to the motion into the positive $x$ direction for electrons entering in the dense plasma).

Regarding the models of the preceding section, where the square momentum deviation $\overline{(\Delta p)^{2}}$ is essentially proportional to powers of the driver amplitudes, the dependence of $D$ on $p$ lies in the typical diffusion time $\delta t$. For the model cases discussed above, the diffusion coefficient can be written as $D=\kappa / p$ for which the key parameters of the model reads

$$
\kappa= \begin{cases}\left(2 / C_{i}\right) a_{d}^{2} / \varepsilon_{p} & \text { for case ' } i, \\ C_{t w} \frac{\pi}{8}\left(\omega_{b} / \omega_{0}\right)^{3}\left(p / \gamma_{0}\right) & \text { for case ' } t w '\end{cases}
$$

wherein $p / \gamma_{0} \simeq 1$ for $p^{2} \gg \gamma_{a}$.

The relation between $\kappa$ and the simulation parameters will be discussed further on in section IIIC 2 and in the context of standard map simulations in section III D.

\section{Solutions to the Fokker-Planck model equation}

From the models assuming stochastic acceleration and heating, a diffusion coefficient that is inversely proportional to $p$ is evident, and also the numerically determined dependence of $D$ shown in Fig. 6 gives evidence for such a dependence of $D \propto 1 / p$ on the momentum. 
On the basis of these results, with $D \sim 1 /|p|$, the form of diffusive term invites to perform a change of variables $(p, t) \rightarrow(u, \tau)$ with $u=p^{3} /(\kappa t)=p^{3} / \tau$ and $\tau=\kappa t$, which yields the FokkerPlanck equation in the form, with $f^{\prime} \equiv \partial f / \partial u$,

$$
f^{\prime \prime} / f^{\prime}=-2 u / 9-1 / 3 \quad \text { for } u>0
$$

from which a solution kernel of the type $\exp \left[-p^{3} /(9 \kappa t)\right]$ results. This leads to a family of solutions - loosely referred as 'similarity solutions ${ }^{9} 67,68-f(u, \tau)$ with the similarity parameter $u=p^{3} /(\kappa t)$, and generated by the function kernel $\exp (-2 u / 9)=\exp \left[-2 p^{3} /(9 \kappa t)\right]$. Indeed, if one seeks for a change of variables $u=p^{\alpha} t^{\beta}$ in order to reduce the FP equation to an ordinary differential equation, the form of diffusive coefficient leads to the relation $\alpha=-3 \beta$ and subsequently $u=$ $\left(t / p^{3}\right)^{\beta}$. The basic choice mentioned above corresponds hence to $\beta \leqslant-1$.

A set of solutions of FP equation can be determined, where we seek for a solution that can explain a cutoff line behavior seen in the distribution. The direct integration of Eq. (12) yields the solution generated by the function kernel $\exp (-2 u / 9)$, and results in the solution denoted below as $f_{3}(u, \tau)$. Other similarity solutions can be found in seeking for solutions of the form $p^{\mu} t^{v} \exp \left[-2 p^{3} /(9 \kappa t)\right]$, which yields exactly two possible combinations $(\mu, v)=(0,-1 / 3)$ and $(\mu, v)=(2,-5 / 3)$.

In the current context, it is preferable to express these solutions as a function of $p$ and of the normalized time $\tau \equiv \kappa t$-instead of the similarity variable $u=p^{3} / \tau-$ which yields functions each with a different time behavior, namely

$$
\begin{aligned}
& f_{1}(p, \tau)=f_{1,0} \tau^{-1 / 3} \exp \left(-2 p^{3} / 9 \tau\right), \\
& f_{2}(p, \tau)=f_{2,0} p^{2} \tau^{-5 / 3} \exp \left(-2 p^{3} / 9 \tau\right), \text { and } \\
& f_{3}(p, \tau)=f_{3,0} \Gamma\left(2 / 3,2 p^{3} / 9 \tau\right),
\end{aligned}
$$

with $\Gamma(. ., .$.$) denoting the incomplete Gamma function, and f_{1,0}, f_{2,0}$, and $f_{3,0}$ as normalization constants. Solution $f_{1}(p, \tau)$ was employed in Ref. ${ }^{38}$ for a Dirac delta function as initial condition. The complementary distributions to the $p d f$ s Eqs. (13-15) defined as $F_{1,2,3} \equiv \int_{p}^{\infty} f_{1,2,3}\left(p^{\prime}, \tau\right) d p^{\prime}$, read

$$
\begin{aligned}
& F_{1}(p, \tau)=6^{-1 / 3} f_{1,0} \Gamma\left(1 / 3,2 p^{3} / 9 \tau\right), \\
& F_{2}(p, \tau)={ }^{3} / 2 f_{2,0} \tau^{-2 / 3} e^{-2 p^{3} / 9 \tau}, \\
& F_{3}(p, \tau)={ }^{1} / 2 f_{3,0}\left[6^{2 / 3} \tau^{1 / 3} e^{-2 p^{3} / 9 \tau}-2 p \Gamma\left(2 / 3,2 p^{3} / 9 \tau\right)\right] .
\end{aligned}
$$


Developing $F_{1}$ and $F_{3}$ for large values of $p^{3} \gg \tau$ yields

$$
\begin{aligned}
& F_{1}(p, \tau)=\frac{3}{2} f_{1,0} \frac{\tau^{2 / 3}}{p^{2}} e^{\frac{-2 p^{3}}{9 \tau}}\left[1-\frac{9}{2} \frac{\tau}{p^{3}}+O\left(\frac{\tau}{p^{3}}\right)^{2}\right] \\
& F_{3}(p, \tau)=\frac{3}{2} f_{3,0} \tau^{1 / 3} e^{\frac{-2 p^{3}}{9 \tau}}\left[\left(\frac{9}{2}\right)^{1 / 3} \frac{\tau}{p^{3}}+O\left(\frac{\tau}{p^{3}}\right)^{2}\right] .
\end{aligned}
$$

All $p d f s$ and distributions have in common that their long range behavior in $p$, for fixed $\tau$, is dominated by the exponentially decreasing function kernel $\exp \left(-2 p^{3} / 9 \tau\right)$. In contrast to $f_{1}$ and $f_{3}$, both monotonically decreasing with $p$ for $p>0$, the solution $p d f f_{2}$ has a time-dependent local maximum at $p=(3 \tau)^{1 / 3}$ before it joins the decrease in $p$ as both other solutions. The $c d f \mathrm{~s}$ hence show three distinguished behaviors in time, $F_{1}$ conserves the mass of the particles with $p>0, F_{1}(p=0, \tau)=$ const, $F_{3}$ increases in time with $\propto \tau^{1 / 3}$ (because $f_{3}(p=0, \tau)=$ const , and $F_{2}$ favors a high-energy tail, but diffuses towards larger values in $p$ and decreases in time like $\propto \tau^{-2 / 3}$.

\section{Application to the simulation}

From the models developed above and from the numerical results, we assume that stochastic heating (or acceleration) explains the observed distributions of energetic electrons entering in the dense plasma. We know however that this type of behavior is only valid where stochastic processes take place, namely in the pre-plasma and in the vicinity of the vacuum-plasma interface skin layer. It has been shown that further inside the dense plasma ${ }^{36}, \mathrm{Ohm}_{\text {-like collisional heating will }}$ dominate, hence a different type of diffusion law.

For this reason it is not adequate to follow (in space and time) the distribution of the electron population that has been accelerated at the interface. This would mean to follow them along their trajectory, where they later on would no longer undergo acceleration in the combined electromagnetic-electrostatic field. We examine the evolution of the distribution further inside in section V.

We therefore have analyzed the electron distribution in strictly the same volume just behind the vacuum plasma interface, i.e. $150<k_{0} x<250$. This distribution witnesses in the positive half-space of the $x-p_{x}$ phase space the time history of potentially re-accelerated particles.

In these distributions deduced from the PIC simulations we observe a clear cutoff like behavior in the electron momentum $p$, particularly pronounced at times $\omega_{0} t<500$ (corresponding to real 


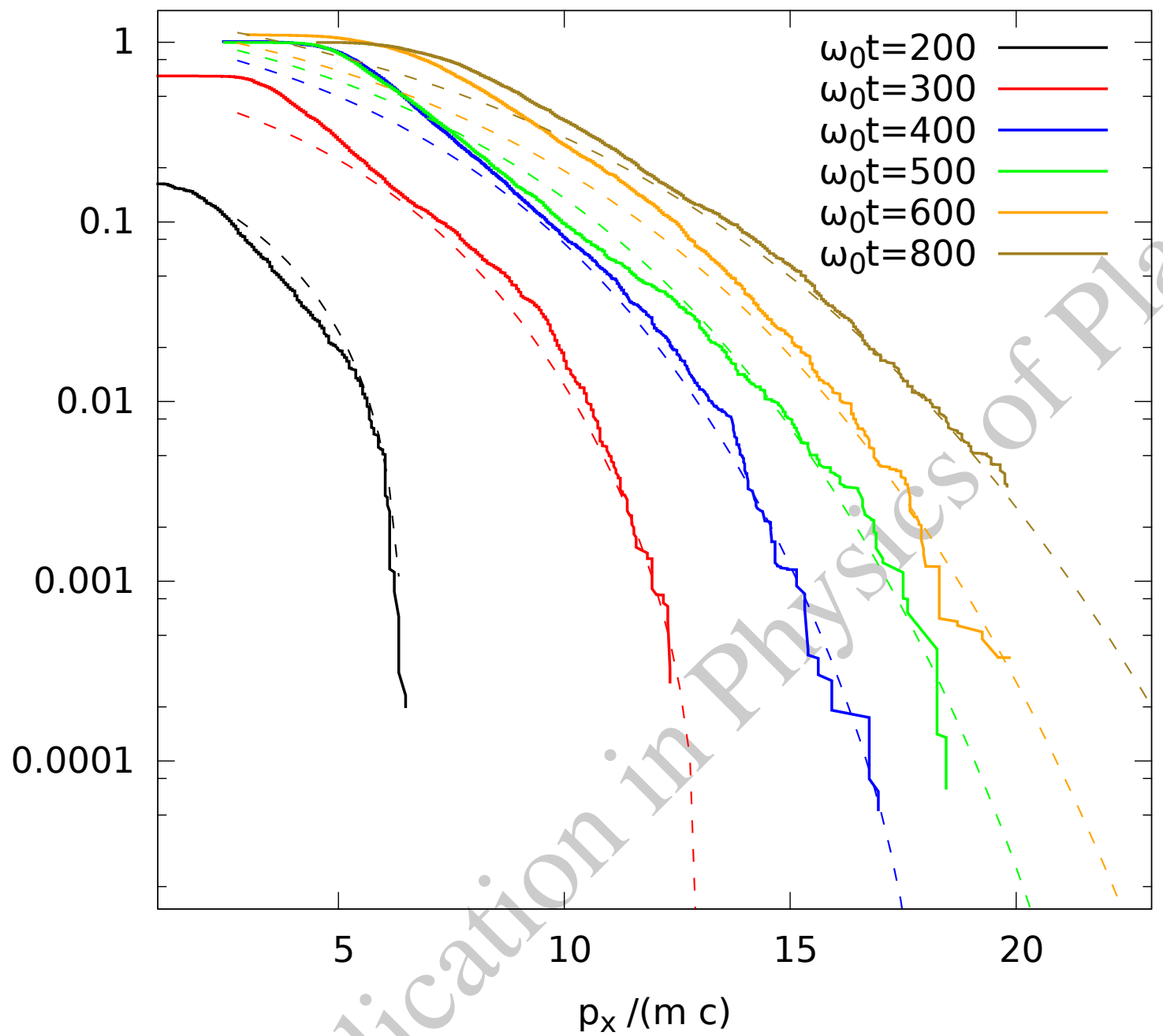

FIG. 7. Distribution of the maxima in the electron momentum for different time instants, $\omega_{0} t=200,300$, 400, 500, 600, and 800 from the simulations (solid lines). The dashed lines show analytic approximations, see text, based on the solution $F_{32}(p, \tau)$, for $\tau=\kappa t$ with $\kappa / \omega_{0}=0.55, c_{32}=7$.

times $t \lesssim 200 \mathrm{fs}$ ), see also Fig. 5. Note that at later times, a clear cutoff can no longer be identified in the simulation due to the limited resolution because of the finite number of particles per cell. Cut-off like behavior for similar configuration has been systematically observed both in MaxwellVlasov and PIC simulations. ${ }^{25,26,28-30,33-35}$ The cutoff in the distribution means that electrons could not be accelerated beyond this value in $p$, or, the probability to find electrons beyond a certain $p$-value drops to zero. It means that electrons cannot attain momenta $p>p_{\max }$. A fast cutoff-like decrease as a function of $p$ cannot be described by a single one of the solutions Eqs. 


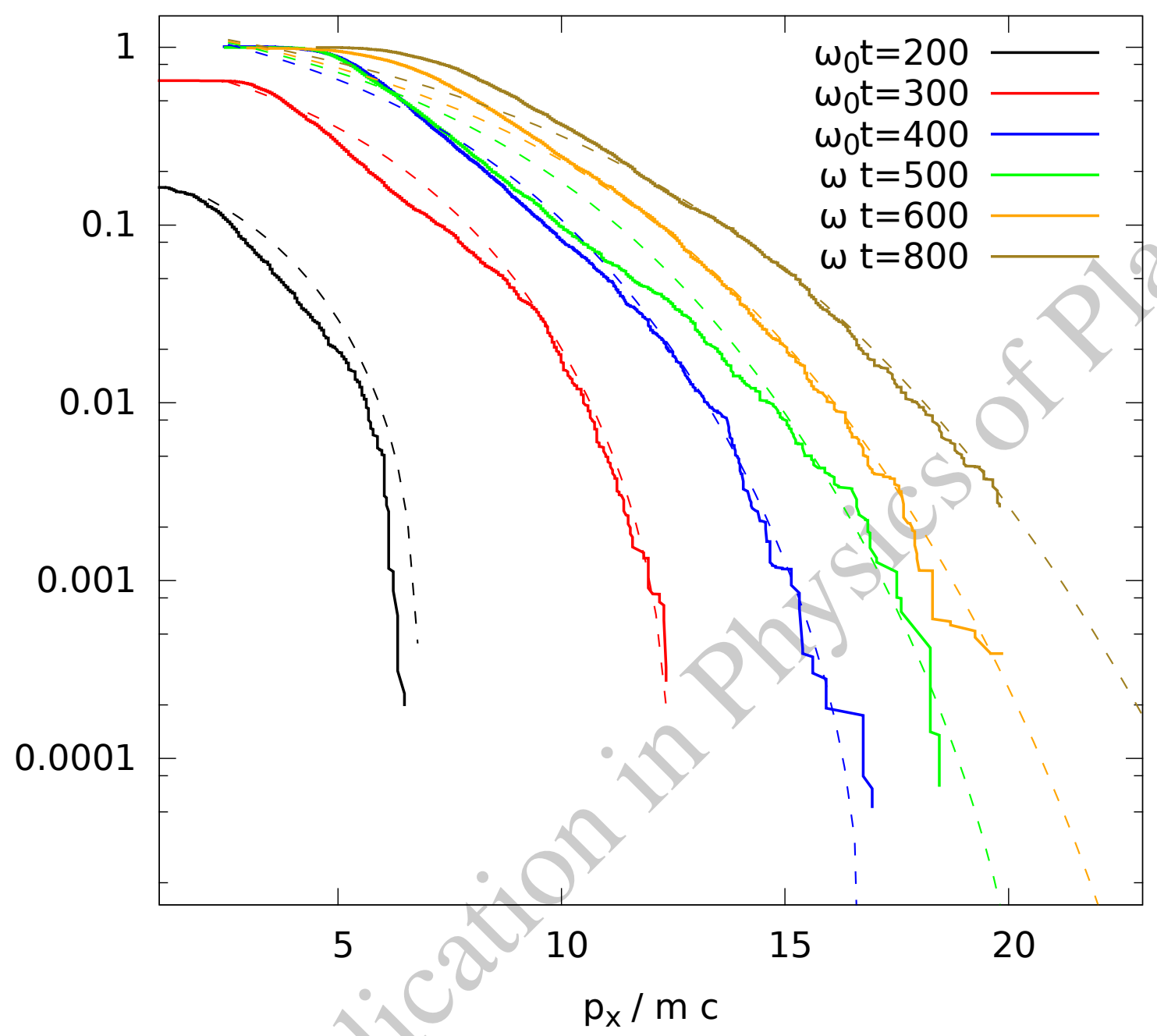

FIG. 8. As in Fig. 7, distribution of the maxima in the electron momentum for different time instants, $\omega_{0} t=200,300,400,500,600$, and 800 from the simulations (solid lines). The dashed lines show analytic approximations, see text, here based on the solution $F_{12}(p, \tau)$, for $\tau=\kappa t$ with $\kappa / \omega_{0}=0.55, c_{12}=3$.

(16-18) derived above from the Fokker-Planck model equation. Observing the value of $p_{\text {contour }}$ where $\bar{F}_{h}$ decrease down to a contour level $\bar{F}_{h}\left(p_{\text {contour }}\right)=$ const, it is evident that this momentum value advances in time like $p_{\text {contour }} \propto \tau^{1 / 3}$.

Via linear combinations between two of the mentioned solutions, however, a cutoff-like decrease, faster than $\exp \left(-2 p^{3} / 9 \tau\right)$, can be described. Relevant combinations of distributions are between $F_{1}$ and $F_{2}$, i.e. $F_{12} \equiv F_{1}-c_{12} F_{2}$, and between $F_{3}$ and $F_{2}$, i.e. $F_{32} \equiv F_{3}-c_{12} F_{2}$, with $c_{12}, c_{32}>0$, which subtracts the contribution of electrons beyond an upper limit in $p$, namely 
$p=p_{\max }$. It means that electrons could not be accelerated to momenta greater than $p_{\max }$ up to this time, such that the distribution is zero beyond this value in $p, F_{12}\left(p>p_{\max }, \tau\right)=0$ or $F_{32}\left(p>p_{\max }, \tau\right)=0$. To be precise, we denote $\bar{F}(p, \tau)=\int_{p}^{p_{\max }}\left[f_{j}\left(p^{\prime}, \tau\right)-c_{j 2} f_{2}\left(p^{\prime}, t\right)\right] d p^{\prime}$, with $j=1$ or 3 in $f_{j}$ and $c_{j 2}$ and where we define $p_{\max }(\tau)$ as the $\sup \left\{p \in R^{+}: \bar{F}_{h}(p)>0\right\}$. By combining the solutions given by $F_{3}$ and $F_{2}$ or $F_{1}$ and $F_{2}$ and using the development Eqs. (19)-(20), we find $\bar{F}(p, \tau)=$

$$
F_{32} \simeq f_{3,0} \frac{e^{-2 p^{3} / 9 \tau}}{\tau^{2 / 3}} \frac{6^{2 / 3} \tau^{2}-c_{32} p^{3}}{p^{3}}, \text { for } p<\frac{6^{2 / 9} \tau^{2 / 3}}{c_{32}^{1 / 3}}
$$

or

$$
F_{12} \simeq \frac{3 f_{1,0}}{2} \frac{e^{-2 p^{3} / 9 \tau}}{\tau^{2 / 3}} \frac{\tau^{4 / 3}-c_{12} p^{2}}{p^{2}}, \text { for } p<\frac{\tau^{2 / 3}}{c_{12}^{1 / 2}}
$$

with the constants $c_{32}=f_{2,0} / f_{3,0}$ and $c_{12}=f_{2,0} / f_{1,0}$.

Clearly, a cutoff-like behavior in $p$ results for $p \rightarrow p_{\max }(\tau)$, where the time-dependent cutoff in $p$ at $p_{\max }$ is proportional to $p_{\max }(\tau) \propto \tau^{2 / 3}$ for both cases Eqs. (21) and (21), more precisely $p_{\max }=c_{32}^{-1 / 3} 6^{2 / 9} \tau^{2 / 3}$ for expression (21), and by $p_{\max }=c_{12}^{-1 / 2} \tau^{2 / 3}$ for (22). Remind that the complementary distribution is defined as $\bar{F}_{h}=0$ beyond the electron momentum $p>p_{\max }(\tau)$. A linear combination between the solutions $F_{1}$ and $F_{3}$ yields a similar cutoff behavior, but the different temporal evolution on the lower energetic part proves that this combination is irrelevant.

In spite of the differences of $F_{32}$ and $F_{12}$ in $p$ far below $p_{\max }$, their behavior is very similar when approaching $p \rightarrow p_{\max }$, namely linear in $p$ for $p<p_{\max }$,

$$
\bar{F}_{h}(p, \tau) \simeq 3 f_{2,0} e^{-2 p_{\max }^{3} / 9 \tau} \tau^{-2 / 3} \frac{p_{\max }(\tau)-p}{p_{\max }(\tau)} .
$$

It is important to remark here, that the level of $\bar{F}_{h}\left(p_{\max }(\tau), \tau\right)$ described by Eqs. (21-22) or (23), decreases in time. This means that the number of particles in the vicinity of the cutoff decreases in time with respect to the ensemble of particles. In PIC simulations with a finite resolution in phase space due to the limited number of particles, this renders the cutoff value $p_{\max }$ unobservable for advanced times. The time dependence of the cutoff, $p_{\max }(\tau)$, depicted in Fig. 5 on the basis of $\bar{F}_{h}$ from our simulation, shows an evolution that fits well with the predicted power law, namely $\propto \tau^{2 / 3}$ up to $\omega_{0} t-140 \simeq 350$, where $\omega_{0} t=140$ is the time instant when the laser arrives at the interface. For $\omega_{0} t>500$, the data extracted from PIC simulations do no longer allow to identify a clear cutoff because of too few particles registered in the distribution.

A good measure for the importance of the most energetic particles close to the cutoff with respect to the hot particle distrtibution is the expectation value $\bar{p}_{h}$, defined as $\bar{p}_{h}=\int_{0}^{p_{\max }} p^{\prime} f_{h}\left(p^{\prime}\right) d p^{\prime}$ 
with $f_{h}$ corresponding to the $c d f$ s Eqs. (21) or (22). This expectation value $\bar{p}_{h}$ also evolves in time, essentially with the power law $\propto \tau^{1 / 3}$ for $\tau \gg 1$, being hence slower than for the cutoff value $p_{\max }(\tau) \propto \tau^{2 / 3}$. The simulation data in Fig. 5 for both the cutoff value in $p$ and for the expectation value $\bar{p}_{h}$ show hence a good agreement with the power laws deduced from the solutions to Eq. (10).

Based on the solutions above, $\bar{F}_{h}=F_{32}$, and $F_{12}$, we have seeked to find the closest agreement with the distributions as shown in Fig. 4 by adapting the parameter $\kappa$, i.e. the conversion from $\omega_{0} t$ to $\tau=\kappa t$, and the normalizing constants $f_{1,0}$ and $f_{2,0}=c_{21} f_{1,0}$ and $f_{1,0}$ and $f_{2,0}=c_{32} f_{3,0}$, respectively. The curves superposed to the PIC-simulation data (here normalized) based on $F_{32}$ in Fig. 7, and on $F_{12}$ in Fig. 8, show very good agreement for a parameter set given by $f_{3,0}=0.1$, $c_{32}=7$, (i.e. $f_{2,0}=7 f_{3,0}$ ) in Fig. 7, as well as, $f_{1,0}=0.8, c_{12}=7$, (i.e. $f_{2,0}=2.95 f_{1,0}$ ) in Fig. 8 . For both cases the value of the key model parameter $\kappa$ proves to converge into a narrow interval $\kappa / \omega_{0} \simeq 0.5 \ldots 0.6$, in agreement with the curve seen in Fig. 6 . This value, $\kappa$, related to the field values via Eq. (11) has here been determined by the best agreement in the distributions $\bar{F}_{h}$.

In Figure 9 we illustrate as well the comparison between PIC-simulation data and the FP solutions $F_{1}(p, \tau)$ when no combination with solution $F_{2}(p, \tau)$ is applied: in this case (and similar for the case based on solution $F_{3}(p, \tau)$, not shown here) no cutoff like behavior is seen, as obvious from Figs. 7 and 8 for times $\omega_{0} t<500\left(\right.$ or $\left.\tau=\left(\kappa / \omega_{0}\right)\left(\omega_{0} t-140\right)<175\right)$.

As already mentioned, for late times, in general the cutoff behavior may become unobservable because of the limited resolution. The time evolution of the highest observable value in $p$ at the lowest contour in $\bar{F}_{h}(p, \tau)$ follows a time law $\propto \tau^{1 / 3}$ corresponding to the dependence of the kernel $\exp \left(2 p^{3} / 9 \tau\right)$ of the similarity solution.

Note that in complement to the time-dependent solutions discussed above, stationary solutions to the FP equation, i.e. for $\partial f / \partial t=0$, yield $p d f$ s with the functional dependence $f(p)=c_{1} p^{2}+c_{2}$ where $c_{1}$ and $c_{2}$ are normalization constants. It is interesting to observe that for $c_{1} / c_{2}<0$ the behavior of the stationary solution corresponds to the near-cutoff behavior of the combined timedependent solutions Eqs. (21-23), derived above.

This would mean that $f(p, t) \sim f\left(p, p_{\max }(t)\right)$ when $d \log \left(p_{\max }(\tau)\right) / d t \ll 1$, so that $f\left(p, p_{\max }(\tau)\right)$ 'almost' satisfies the stationary $F P$ equation for sufficiently small $\partial f / \partial t \simeq 0$, i.e. $\partial_{p p} f \simeq$ $(1 / p) \partial_{p} f$

We now compare the coefficients deduced from the simulation with coefficients expected from the models : 


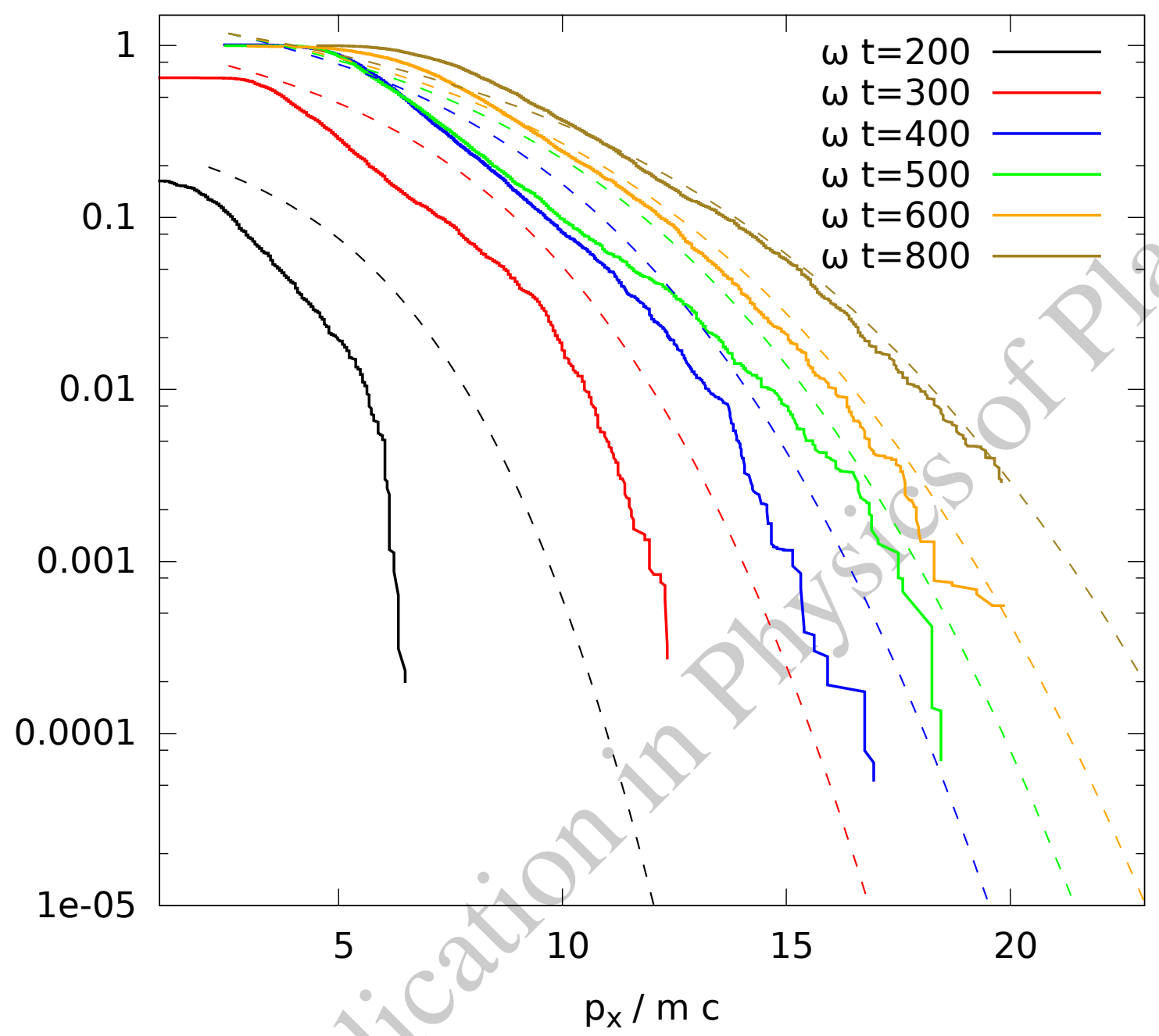

FIG. 9. As in Fig. 7, distribution of the maxima in the electron momentum for different time instants, $\omega_{0} t=200,300,400,500,600$, and 800 from the simulations (solid lines). The dashed lines show analytic approximations, see text, here based on the solution without cutoff behavior, $F_{1}(p, \tau)$, for $\tau=\kappa t$ with $\kappa / \omega_{0}=0.51$.

(" $i$ ") In the vicinity of the interface, the relevant model parameters are the electric field strength $\varepsilon_{p}$ and the driver amplitude $a_{d}$. The peak value of the electric field in the layer close to the interface is in the range $\varepsilon_{p} \simeq 0.2 \ldots 0.26$, without being spatially homogeneous. The value of $a_{d}$ can also be estimated from the ponderomotive force (roughly $a_{d} \sim a_{\perp, 0}^{2} /\left(2 \omega_{0} / \omega_{p}\right)$, that taken at $k_{0} x \simeq 136$ yields $a_{d} \sim 0.1 \ldots 0.3$. Independent of the value of $\kappa$ that results, it is important to remark that the driver field characterized by $a_{d}$ is too much localized in $x$, namely in the very narrow skin layer. 
For this reason multiple acceleration events via the interplay between the motion in the driver and the electrostatic field are very unlikely for already energetic electrons. This process can hence be excluded in the current context to explain the temporal evolution of the ultra-relativistic electron distribution.

(" $\left.t w^{\prime \prime}\right)$ In the pre-plasma in presence of the standing wave, the parameter $\kappa$ can be evaluated from Eq. (11), i.e. $\kappa=C_{t w}(\pi / 8)\left(\omega_{b} / \omega_{0}\right)^{3}$. To be consistent with the numerically determined value $\kappa \sim 0.6$ mentioned above would require $\omega_{b} / \omega_{0} \simeq 1.2$. With the relation between the bounce frequency and the field strength from Eq. (4), for $a_{\perp, 0}=3$, equivalently the value of $\sqrt{r} \simeq 0.4$ would result. This value is by a factor of $1 / 4$ below the value of the reflectivity observed, namely of $r_{\text {PIC }}=0.82$ in field (from PIC simulations, $r_{\text {PIC }}^{2}=70 \%$ in intensity). Higher harmonics in the reflected light could however explain this discrepancy: inspection of the electromagnetic field profiles in $x$, along the axis of the incoming and reflected laser beams, show the presence odd higher harmonics to the spatial frequency $2 k_{0}$ in the ponderomotive force. Field amplitudes reach values of the order of $a_{\perp, \max }=5 \ldots 6.5$ where peaks due to higher harmonics are clearly in excess of a sinusoidal shape of the fundamental only, for which total amplitudes of $(1+r) a_{\perp, 0} \simeq 3.6<a_{\perp, \max }$ would result.

The coefficient $\kappa$ allows us to relate the cutoff seen in the electron momentum to the laser pulse parameters, i. e. via $p_{\max } \propto\left(\omega_{b} / \omega_{0}\right)^{2}\left(\omega_{0} t\right)^{2 / 3}$ where the bounce frequency in the standing wave $\omega_{b}$ is related to the laser intensity, Eq. (4), resulting in the scaling $p_{\max } \propto a_{0}^{2}\left(\omega_{0} t\right)^{2 / 3}$. Similarly, for the value of the expectation value in $p$ of the energetic electrons, $\bar{p}_{h}$, one obtains the weaker scaling $\bar{p}_{h} \propto a_{0}\left(\omega_{0} t\right)^{1 / 3}$.

\section{Standard map simulation}

Based on the standard map model discussed in section III A, we have performed simulations of the set of equations

$$
\begin{gathered}
p_{x, n+1}=p_{x, n}+C_{t w}\left(\omega_{b} / \omega_{0}\right) \sin \left(\phi_{n}\right) \\
\phi_{n+1}=\phi_{n}+2 C_{t w}\left(\omega_{0} / \omega_{b}\right) p_{x, n+1}
\end{gathered}
$$

which is equivalent to Eqs. (6)-(7), and in which, via $p_{x}=P\left(\omega_{b} / \omega_{0}\right) /\left(2 C_{t w} \pi\right)$ the parameters are $C_{t w}$ and $\omega_{b} / \omega_{0}$. To do so, we have run the map over 50 iterations for 50000 particles in order to determine histograms in $p_{x}$ of the particles as a function of time, where the time is associated with 


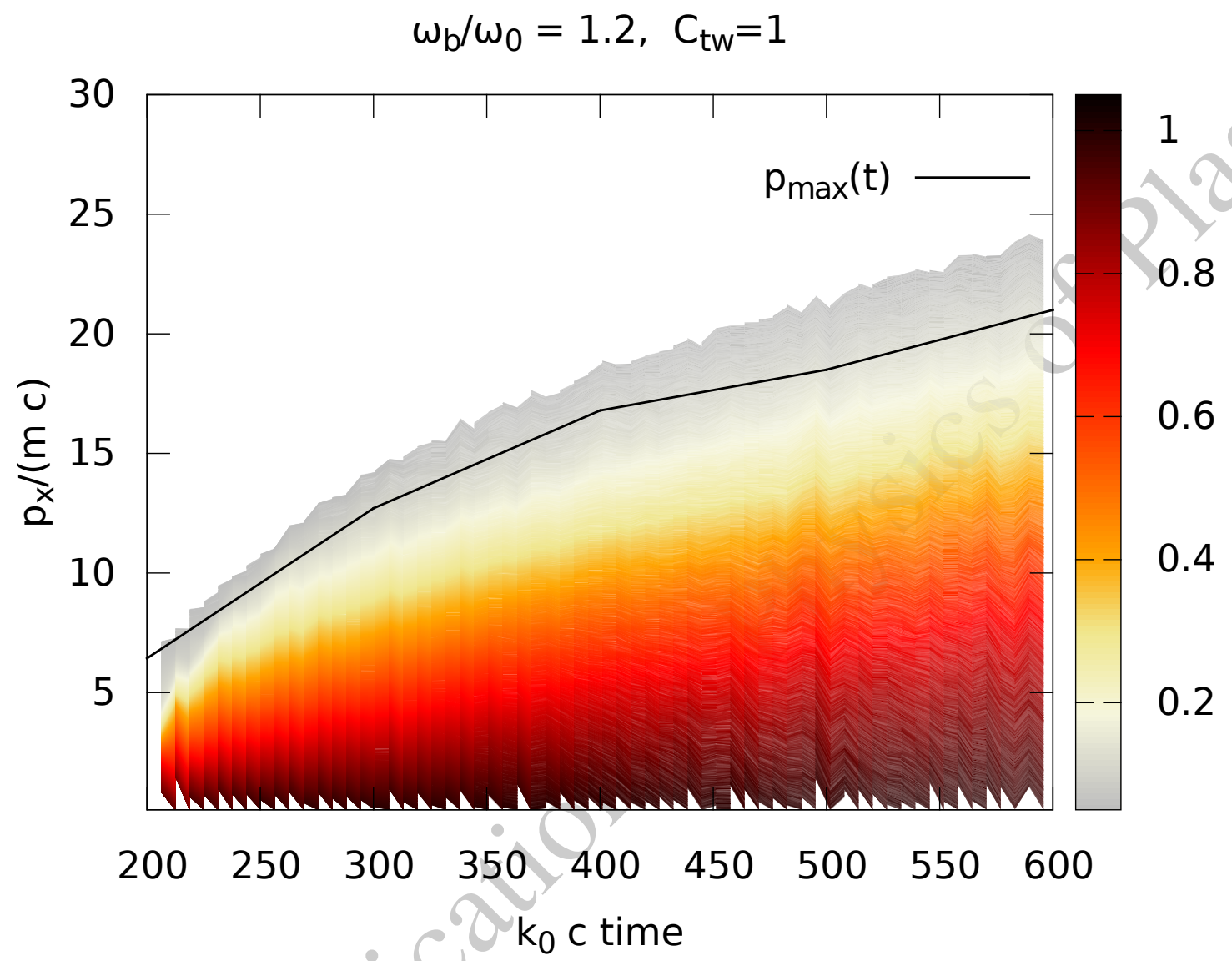

FIG. 10. Contour map of the complementary distribution $\bar{F}_{\text {map }}(p, t)$, as a function of the electron momentum and time, derived from a simulation following the standard map, Eqs. (24-25), for 50 iterations and 50000 particles. Parameters $\omega_{b} / \omega_{0}=1.2$ and $C_{t w}=1$. The curve for $p_{\max }(t)$ from Fig. 5, marked with line, is reported in this plot.

the phase $\phi_{n}$ by not applying the modulo with $2 \pi$, hence $\phi_{n} \equiv\left(2 k_{0} x\right)_{n} \simeq 2 k_{0} c t_{n}$.

The standard map simulations were initialized using a uniform distribution in the initial particle phase in the interval $[0,2 \pi)$. For the initial momenta of the particles we have varied the interval between $\left[-1 m_{e} c,+1 m_{e} c\right]$ and $\left[-3 m_{e} c,+3 m_{e} c\right]$ without observing essential differences. After all iterations (index $n$ ), the obtained data pairs $\left\{k_{0} c t_{n}, p_{x, n}\right\}_{j}$ for the 50000 particles (index $j$ ) were first ordered in time, and then again, for each time interval, in the magnitude of $\left|p_{x}\right|$. The ordering 
from the latter operations yields hence histograms which can be considered as particle distributions $\bar{F}_{\text {map }}\left(p_{x}, t\right)$.

The best agreement between standard map simulations and the temporal evolution of the cutoff contour, see Fig. 5, is found for the interval $\omega_{b} / \omega_{0}=1 \ldots 1.5$, which is consistent with the conclusion from section III C 2. The result from such a simulation with $C_{t w}=1$ and $\omega_{b} / \omega_{0}=1.2$ is shown in Fig. 10. Note the time shift $k_{0} c t \simeq 140$ between the standard map and the PIC simulations due to the retarded arrival of the light wave at the interface $k_{0} x \simeq 140$.

It is remarkable that the distribution extracted from the standard map simulation, although not retaining all the complexity of the physical effects described in the PIC simulations, recovers essential features of the acceleration effects in the pre-plasma and at the interface.

\section{EXTREME THEORY}

As explained in section II we have analyzed the electron distribution in strictly the same volume just behind the vacuum plasma interface, i.e. $150<k_{0} x<250$. Each $x_{i}$ of the numerical resolution is recorded for $i=1$ to $\mathscr{N}$. Since the electron bursts are injected periodically in time, $\pi / \omega_{0}$, they appear hence in regular spatial intervals of the length $c \times \pi / \omega_{0}$. We denote them as $\mathscr{I}_{1}, \ldots, \mathscr{I}_{n}, \ldots, \mathscr{I}_{N}$, with $\mathscr{I}_{n}=\left[x_{0}+n \pi c / \omega_{0}, x_{0}+(n+1) \pi c / \omega_{0}\right)$. The variables $p_{\max }\left(x_{n}\right)$ with $x_{n} \in \mathscr{I}_{n}$, for $n=1, \ldots, N$ form a sequence of independent, equally distributed variables with distribution $\bar{F}_{h}(p)$. Moreover, the distribution $\bar{F}(p)$ and $\bar{F}_{h}(p)$ are tail-equivalent. We hence define the sequence $M_{n}=\max \left\{p_{\max }\left(x_{1}\right), p_{\max }\left(x_{2}\right), \ldots, p_{\max }\left(x_{n}\right)\right\}$ with $x_{n} \in \mathscr{I}_{n}$. for which we examine the limit law.

We recall here from Ref. 40 the fundamental result of extreme value theory which we shall use further on for comparison with our numerical results. Consider a random variable $p$, following a distribution $F$, with its corresponding density $f$, and with the complementary distribution function $\bar{F}(p)=C\left(p_{F}-p\right)^{\alpha}$, having the finite right endpoint $p_{F}(=\sup \{p \in R: F(p)<1\})$, and with an exponent $\alpha>0$. One can show that $F$ belongs then to the maximum domain of attraction of the Weibull law. This means that the sequence of maxima $M_{n}=\max \left(p_{1}, \ldots, p_{n}\right)$ corresponding to the sequence of independent and equi-distributed random variables $p_{i}$ with a common distribution $F$, adequately recentred and rescaled, converges in distribution to the Weibull law:

$$
P\left[(n C)^{1 / \alpha}\left(M_{n}-p_{F}\right)<p\right] \longrightarrow \exp \left(-(-p)^{\alpha}\right),
$$

with $C$ as the scaling constant, and $n$ denoting the number of elements in the sequence. To deal 
with our distribution $F$ from Eq. (21), we show that they have the same behavior as the model distribution $F(p)=1-C\left(p_{F}-x\right)^{\alpha}$ in that they satisfy 'von Mises' condition ${ }^{40}$ :

$$
\lim _{p \uparrow p_{F}} \frac{\left(p_{F}-p\right) f(p)}{\bar{F}(p)}=\alpha>0,
$$

which implies that $F$ belongs to the maximum domain of attraction of the Weibull law $\Psi_{\alpha}=$ $\exp \left(-(-p)^{\alpha}\right)$. This limit (von Mises) yields, for our prototype distribution $\bar{F}(p, \tau)=\exp \left(-2 p^{3} / 9 \tau\right) p^{-1} \tau^{-2 / 3}\left(p_{\mathrm{r}}^{3}\right.$ $p^{3}$ ), whose behavior near $p_{\max }$ is governed by $\sim 2 p_{\max }\left(p_{\max }-p\right)$ such that we obtain $\lim _{p \uparrow p_{\max }}\left(p_{\max }-\right.$ $p) f(p) / \bar{F}(p)=1$, by replacing $p_{F}$ in Eq. (27) by $p_{F}=p_{\max }$. The exponent in Eq. (27) is hence $\alpha=1$ insuring convergence of the law of $M_{n}$ toward the Weibull law $\Psi_{1}=\exp (-(-p))$. Note that as $\bar{F}_{h}(p)$ and $\bar{F}(p)$, as defined in section II are tail-equivalent, i.e. the same result is valid for both $\bar{F}_{h}(p)$ and $\bar{F}(p)$. Extreme theory shows that for an exponent in the power law $\alpha=1$, the limit law for extreme values in the distribution, hence the distribution of the most energetic electrons, is a Weibull distribution given by

$$
\bar{F}(p) \sim 1-\left[1-n^{-1}\left(p_{\max }-p\right) / p_{\Delta}\right]^{n},
$$

for a finite number of $n$ independent values measured for the most energetic electrons, yielding asymptotically for $n \rightarrow \infty$

$$
\bar{F}(p) \sim 1-e^{\left(p-p_{\max }\right) / p_{\Delta}}
$$

From the reference simulation we have hence determined histograms of the maxima in the peak values in the electron bursts, as for the example shown in the phase space, denoted as '+' in Fig. 3. These histograms are obtained on the same data as used for Figs. 4 and for time instants $\omega_{0} t=$ 200, 300. The result, in linear scale, is shown in Fig. 11, for times $\omega_{0} t=200,300,400$, and 500 . Superposed to the numerically determined distribution are the distribution following the Weibull limit law given by Eq. 29 for which at each time $t$ the cutoff value $p_{\max }(t)$ is used. The limit law predicts hence a linear decrease of the extreme value distributions in the neighborhood of $p=p_{\max }$ for $p \leq p_{\max }$ beyond which $\bar{F}$ is zero. This is well reproduced by Eq. (29) and best agreement is achieved when also the interval $p_{\Delta}$ assumes $p_{\Delta} \simeq p_{\max }(t)$. For later times, beyond $\omega_{0} t \sim 400$, and illustrated for $\omega_{0} t=500$, the agreement with the limit law fails because the cutoff in the numerically deduced electron distributions is no longer well resolved, as already mentioned earlier, see Fig. 5.

The agreement found between the numerically determined distributions and the extreme value theory is hence a clear signature of the cutoff behavior described by the solutions to the FokkerPlanck equation associated to the stochastic acceleration model. 


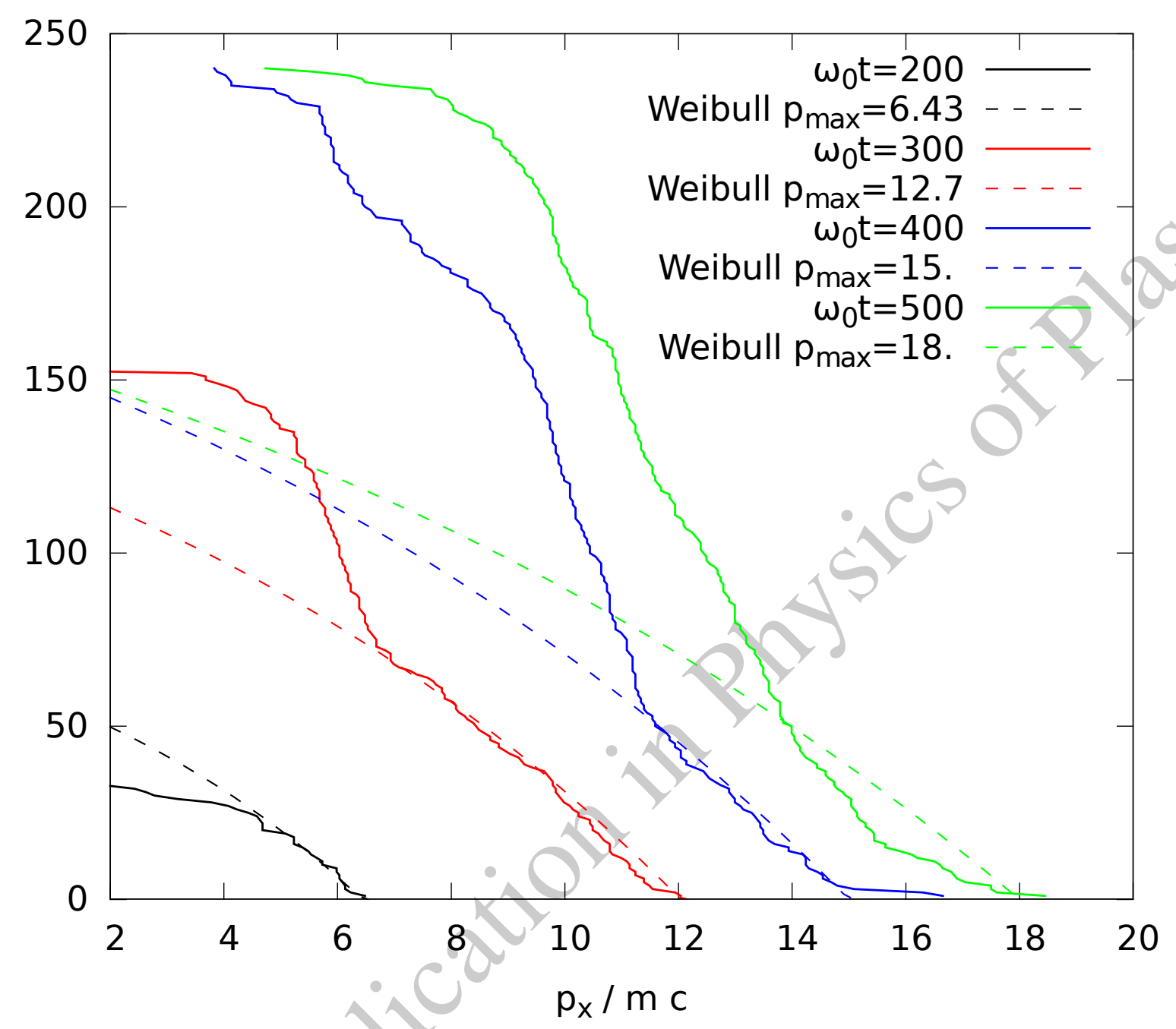

FIG. 11. Complementary distributions, $\hat{F}\left(p_{x}\right)$ (solid lines) of the maxima in the electron momentum (multiplied with the number of maxima counted), sampled over the maxima in $p_{x}$ in the bursts of the phase space $150<k_{0} x<250$, behind the vacuum plasma interface. The functions fitted to the data values (dashed lines), for the different times, $\omega_{0} t=200,300,400$, see legends, correspond to Weibull distributions in the tail, $p_{x} \rightarrow p_{\max }$, with $\hat{F} \sim \exp \left[\left(p_{x}-p_{\max }(t)\right) / p_{\Delta}(t)\right]$, with $p_{\Delta}=p_{\max }$ and by using the value of $p_{\max }(t)$ for to the different time instants $t$. For $\omega_{0} t=500$ the cutoff type behavior is no longer resolved in the PIC simulations, see text.

\section{DISCUSSION AND CONCLUSIONS}

We have studied the evolution of the distibution function of electrons that penetrate into a dense plamsa after being accelerated by an intense laser wave in the vicinity of the interface between vac- 
uum and plasma. Our analysis is based on a reference simulation in the relativistic laser intensity regime. The evolution seen in the distribution of the most energetic electrons inside the plasma does not correspond to the momenta expected from a single laser-acceleration event, but can be explained by stochastic acceleration. We find that the electron distribution function in the layer behind the interface shows a cutoff in the particle momentum and consequently the energy. The cutoff in momentum, $p_{\max }$, evolves in time.

In spite of the complex electron acceleration mechanisms in the vicinity of the solid-density interface, the essential physics in play proves to be governed by models for stochastic acceleration. Electrons are ejected from the layer close to the solid-density plasma interface in both directions. Those energetic electrons that enter into the standing wave, formed by the incident laser wave and the reflected light, are easily found to be in a parameter regime for stochastic motion for relativistic laser intensities. Their motion in this standing wave, where electrons can also be reversed in their direction, leads eventually to a net increase in their energy. The relevance of the model based on stochastic acceleration in a standing wave pattern relies on a not too small reflectivity coefficient $r_{\text {PIC }}$ of the incident laser wave.

From the analysis of the distribution functions, we show that the stochastic motion leads to diffusion in momentum space which can be described by a certain class of solutions to the FokkerPlanck equation. Numerical data show, in consistence with the model for the Fokker-Planck equation, that the diffusion coefficient is inversely proportional to the electron momentum in $x$ dimension, i.e. normal to the interface. We find that relevant solutions to the model show a cutoff behavior in the electron momentum that follows a power law behavior in time $p_{\max }(t) \propto a_{0}^{2}\left(\omega_{0} t\right)^{2 / 3}$. The expectation value in momentum of the energetic electron, $\bar{p}_{h}$ also evolves in time with a power-law like behavior, but with a smaller exponent, namely $\bar{p}_{h}(t) \propto a_{0}\left(\omega_{0} t\right)^{1 / 3}$. For the cutoff value $p_{\max }$ as well as for the expectation value $\bar{p}_{h}$, the above mentioned dependences in terms of power laws dependence are seen in the reference PIC simulation, as illustrated in Fig. 5. Note that the cutoff in the distribution, at $p_{\max }(t)$, is more pronounced in the early part of the laser pulse. Later on, the phase space resolution in PIC simulations may no longer be sufficient to observe a clear cutoff, while this should still be possible in Vlasov-Maxwell simulations.

The model used here to interpret the simulation results essentially relies on the description in a single spatial dimension, namely in the direction along the laser propagation. The physics of the interaction in the vicinity of the vacuum-plasma density interface is, however, extremely complex. Multi-dimensional aspects in this context have already been discussed in previous work. ${ }^{27,30}$ The 
strength and the structure of the laser field in the vicinity of the interface strongly depends on the geometry of the interaction, such as polarisation, the incidence angle, ${ }^{24}$ but also on the presence of surface structures. 35,69

Indeed, an important factor for the stochastic acceleration is the number of electrons that are ejected from the interface into the standing wave, resulting from the superposition of incident and reflected light fields. In order to examine the role of the geometry as well as the plasma density profile, we have performed simulations in one- (1D) and two-dimensional (2D) geometry, and with different density gradients. In 1D simulations, we observe that the density gradient steepens considerably stronger than in the same case in $2 \mathrm{D}$, which has the result that in $1 \mathrm{D}$ only few electrons are ejected from the interface, much less than in the $2 \mathrm{D}$ case. We have furthermore performed a simulation with an initially step-like profile (i.e. an infinitely steep gradient at $t=0$ ). This case produces essentially fast electrons with $p_{x} /\left(m_{e} c\right)>a_{0}$ entering into the dense plasma. The main acceleration process takes place at the interface, i.e. in the skin layer, mostly via a single acceleration event. Only an insignificant number of particles undergoes the stochastic acceleration in a standing wave. However, in 2D simulations with finite plasma density gradients, the number of electrons ejected into the standing wave in front of the interface is drastically higher compared to the corresponding 1D case. Similar observations were reported in Ref. 30.

While our modelling does not take into account filamentary structure inside the standing wave pattern, it is evident from the simulations that the effect of laser light filamentation (for 2(3)D cases), upcoming with increasing laser intensity, counts for the number of electrons that are stochastically accelerated, namely in two ways: (i) the electrons that are directly injected in the dense plasma attain higher energies (with respect to a plane wave case, as in 1D) due to the locally increased intensities at the interface; this has the consequence that the highest momentum ovserved from directly injected electrons depends on the peak amplitude of the filamented field; (ii) the number of electrons ejected in the superposed light fields augments with filamentation such that also the electron population that is stochastically accelerated increases as well. We have, however, not investigated in more detail how strong the stochastic acceleration is affected by the upcoming filamentation when increasing the laser intensity beyond the value of the reference simulation. We can conclude from our simulation studies that 2D physics plays an essential role for the number of electrons entering the standing wave formed by the light fields. The principal process that explains stochastic heating of the fastest particles can, however, be described by the 1D model for electron motion in a standing wave. Concerning now the time evolution of the distribu- 
tion $\bar{F}_{h}\left(p_{x}, t\right)$ described by our model for energetic electrons entering into the dense plasma: it is the number and the distribution of electrons that enter the standing electromagnetic wave that determines the evolution of the cutoff in the electron momentum $p_{\max }(t)$, namely via the coefficients $\left(f_{i, 0}, i=1,2,3\right)$ to the solutions Eqs. (13)-(23).

We finally discuss the evolution of the electron distribution further inside the plasma. There, the electrons will no longer be under the influence of the forces that are present in the skin layer. For this reason, and again on the basis of the simulation results, we have determined how the distribution evolves when following the trajectory of energetic electrons further inside the dense plasma. The particles in the tail of the distribution move almost all with the speed of the light, so that one can examine the evolution of $\bar{F}_{h}\left(p_{x}, t\right)$ determined in the vicinity of $k_{0} x=150$, at times $t+\delta t$ at $k_{0}(x+c \delta t)$. This is illustrated in Fig. 12, where the distribution $\bar{F}_{h}$ is followed for two times, $\omega_{0} t=200$ and 400 taken over the interval $150<k_{0} x<250$, and then for $\omega_{0}(t+\delta t)$ over the intervals $150+c \delta t<k_{0} x<250+\delta t$, with $\delta t=50,100,200$, and 300. For both cases shown, the cutoff value seen in $p_{x}$ does not considerably evolve, and in particular, a sharp cutoff shape is maintained. In the case of the distribution observed at $\omega t=400$ in $150<k_{0} x<250$, the cutoff value slightly decreases, most probably due to momentum transfer in the $p_{y}$-component, and due to the upcoming return current modifying the trajectory of electrons that initially entered with a strongly dominant $p_{x}$ component.

The distribution of those electrons accelerated at the vacuum-plasma interface is of importance for ion acceleration processes at the rear side of a target with finite width, and it is of interest for the models describing ion acceleration. ${ }^{70-83}$ The evolution shown in Fig. 12, illustrating how the most energetic electron penetrate into the dense plasma, are therefore of particular relevance for relatively thin target foils for which the cutoff in the electron momentum remains 'preserved' till the arrival of the fastest electrons at the rear face.

\section{ACKNOWLEDGEMENT}

We thank Patrick Mora, Wojciech Rozmus, Jean-Marcel Rax, and Peter Mulser for useful discussions. This work was possible thanks to access to the high performance computing resources from GENCI-IDRIS, project nos. 2012/2014-056044 and A0060500573. 


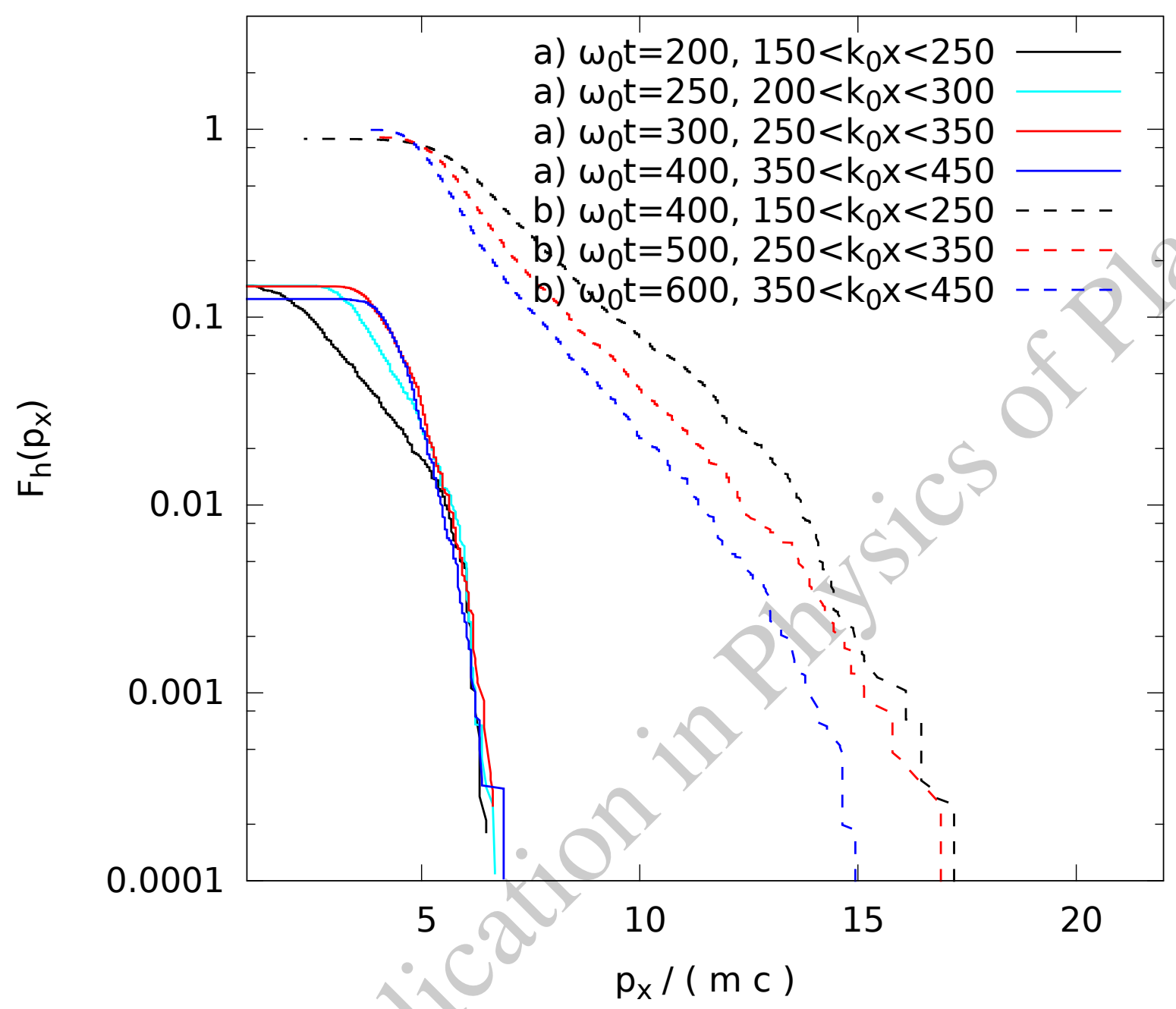

FIG. 12. Complementary distribution $\bar{F}_{h}\left(p_{x}, t\right)$, as Fig.7, from PIC simulations as a function of time, but deduced from particles in a comoving window with $\sim c$, as indicated in the legend: the left-side group of curves (solid lines) follows the early time behavior, a), starting with $\omega_{0} t=200$ in the spatial window $150<k_{0} x<250$ followed up with $\omega_{0} t=250,300$, and 400 , the latter one in the window $350<k_{0} x<450$; the right side curves (dashed lines), b), show the behavior starting at $\omega_{0} t=400$ in the window $150<k_{0} x<250$, followed by $\omega_{0} t=500$, and 600 , the last one in the window $350<k_{0} x<450$.

\section{REFERENCES}

${ }^{1}$ S. C. Wilks, W. L. Kruer, M. Tabak, and A. B. Langdon, Phys. Rev. Lett. 69, 1383 (1992).

${ }^{2}$ S. C. Wilks, Physics of Fluids B: Plasma Physics 5, 2603 (1993).

${ }^{3}$ G. Malka and J. L. Miquel, Phys. Rev. Lett. 77, 75 (1996). 
${ }^{4}$ J. Denavit, Phys. Rev. Lett. 69, 3052 (1992).

${ }^{5}$ E. L. Clark, K. Krushelnick, J. R. Davies, M. Zepf, M. Tatarakis, F. N. Beg, A. Machacek, P. A. Norreys, M. I. K. Santala, I. Watts, and A. E. Dangor, Phys. Rev. Lett. 84, 670 (2000).

${ }^{6}$ K. Krushelnick, E. L. Clark, M. Zepf, J. R. Davies, F. N. Beg, A. Machacek, M. I. K. Santala, M. Tatarakis, I. Watts, P. A. Norreys, and A. E. Dangor, Physics of Plasmas 7, 2055 (2000).

${ }^{7}$ R. A. Snavely, M. H. Key, S. P. Hatchett, T. E. Cowan, M. Roth, T. W. Phillips, M. A. Stoyer, E. A. Henry, T. C. Sangster, M. S. Singh, S. C. Wilks, A. MacKinnon, A. Offenberger, D. M. Pennington, K. Yasuike, A. B. Langdon, B. F. Lasinski, J. Johnson, M. D. Perry, and E. M. Campbell, Phys. Rev. Lett. 85, 2945 (2000).

${ }^{8}$ M. Hegelich, S. Karsch, G. Pretzler, D. Habs, K. Witte, W. Guenther, M. Allen, A. Blazevic, J. Fuchs, J. C. Gauthier, M. Geissel, P. Audebert, T. Cowan, and M. Roth, Phys. Rev. Lett. 89, 085002 (2002).

${ }^{9}$ S. Bulanov, T. Esirkepov, V. Khoroshkov, A. Kuznetsov, and F. Pegoraro, Physics Letters A 299, 240 (2002).

${ }^{10}$ L. O. Silva, M. Marti, J. R. Davies, R. A. Fonseca, C. Ren, F. S. Tsung, and W. B. Mori, Phys. Rev. Lett. 92, 015002 (2004).

${ }^{11}$ S. Kar, M. Borghesi, S. V. Bulanov, M. H. Key, T. V. Liseykina, A. Macchi, A. J. Mackinnon, P. K. Patel, L. Romagnani, A. Schiavi, and O. Willi, Phys. Rev. Lett. 100, 225004 (2008).

${ }^{12}$ T. Schlegel, N. Naumova, V. T. Tikhonchuk, C. Labaune, I. V. Sokolov, and G. Mourou, Physics of Plasmas 16, 083103 (2009).

${ }^{13}$ A. Macchi, M. Borghesi, and M. Passoni, Rev. Mod. Phys. 85, 751 (2013).

${ }^{14}$ M. Borghesi and A. Macchi, "Laser-driven ion accelerators: State of the art and applications," in Laser-Driven Particle Acceleration Towards Radiobiology and Medicine, edited by A. Giulietti (Springer International Publishing, Cham, 2016) pp. 221-247.

${ }^{15}$ J. Sanz, A. Debayle, and K. Mima, Phys. Rev. E 85, 046411 (2012).

${ }^{16}$ A. Debayle, J. Sanz, L. Gremillet, and K. Mima, Physics of Plasmas 20, 053107 (2013).

${ }^{17}$ A. Debayle, J. Sanz, and L. Gremillet, Phys. Rev. E 92, 053108 (2015).

${ }^{18}$ P. Mulser and D. Bauer, High Power Laser-Matter Interaction (Springer, Heidelberg, 2010) pp. p. 355-359.

${ }^{19}$ S. V. Bulanov, T. Z. Esirkepov, M. Kando, S. S. Bulanov, S. G. Rykovanov, and F. Pegoraro, Physics of Plasmas 20, 123114 (2013).

${ }^{20}$ J. T. Mendonça, Phys. Rev. A 28, 3592 (1983). 
${ }^{21}$ D. Bauer, P. Mulser, and W. H. Steeb, Phys. Rev. Lett. 75, 4622 (1995).

${ }^{22}$ Z.-M. Sheng, K. Mima, Y. Sentoku, M. S. Jovanović, T. Taguchi, J. Zhang, and J. Meyer-ter Vehn, Phys. Rev. Lett. 88, 055004 (2002).

${ }^{23}$ Z.-M. Sheng, K. Mima, J. Zhang, and J. Meyer-ter Vehn, Phys. Rev. E 69, 016407 (2004).

${ }^{24}$ H. Ruhl, J. Opt. Soc. Am. B 13, 388 (1996).

${ }^{25}$ T. Liseykina, P. Mulser, and M. Murakami, Physics of Plasmas 22, 033302 (2015).

${ }^{26}$ R. Mishra, Y. Sentoku, and A. J. Kemp, Physics of Plasmas 16, 112704 (2009).

${ }^{27}$ A. J. Kemp, Y. Sentoku, and M. Tabak, Phys. Rev. E 79, 066406 (2009).

${ }^{28}$ M. Sherlock, Physics of Plasmas 16, 103101 (2009).

${ }^{29} \mathrm{M}$. Sherlock and W. Rozmus, "Vlasov simulations of intense $10^{19} \mathrm{~W} \mathrm{~cm}^{-2}$ laser interactions with overdense plasma of varying scale-length," CLF Annual Report 2007-2008 (Rutherford Appleton Laboratory, 2007-2008).

${ }^{30}$ J. May, J. Tonge, F. Fiuza, R. A. Fonseca, L. O. Silva, C. Ren, and W. B. Mori, Phys. Rev. E 84, $025401(2011)$.

${ }^{31}$ A. P. L. Robinson, A. V. Arefiev, and D. Neely, Phys. Rev. Lett. 111, 065002 (2013).

${ }^{32}$ G. E. Kemp, A. Link, Y. Ping, D. W. Schumacher, R. R. Freeman, and P. K. Patel, Physics of Plasmas 20, 033104 (2013), https://doi.org/10.1063/1.4794961.

${ }^{33}$ A. Sorokovikova, A. V. Arefiev, C.McGuffey, B. Qiao, A. P. L. Robinson, M. S. Wei, H. S. McLean, and F. N. Beg, Phys. Rev. Lett. 116, 155001 (2016).

${ }^{34}$ S. Kojima, M. Hata, N. Iwata, Y. Arikawa, A. Morace, S. Sakata, S. Lee, K. Matsuo, K. F. Farley Law, H. Morita, Y. Ochiai, A. Yogo, H. Nagatomo, T. Ozaki, T. Johzaki, A. Sunahara, H. Sakagami, Z. Zhang, S. Tosaki, Y. Abe, J. Kawanaka, S. Tokita, M. Nakai, H. Nishimura, H. Shiraga, H. Azechi, Y. Sentoku, and S. Fujioka, ArXiv e-prints (2018), arXiv:1803.02514 [physics.plasm-ph].

${ }^{35}$ C. Riconda, M. Raynaud, T. Vialis, and M. Grech, Physics of Plasmas 22, 073103 (2015).

${ }^{36}$ A. Héron and J. C. Adam, Physics of Plasmas 22, 072306 (2015).

${ }^{37}$ Further inside, where the fields in the skin layer have no influence at all, the essential physical effects change, see $^{36}$, and angular momentum transfer arises.

${ }^{38}$ S. V. Bulanov, A. Yogo, T. Z. Esirkepov, J. K. Koga, S. S. Bulanov, K. Kondo, and M. Kando, Physics of Plasmas 22, 063108 (2015).

39J. M. Rax, Physics of Fluids B: Plasma Physics 4, 3962 (1992). 
${ }^{40}$ P. Embrechts, C. Klüppelberg, and T. Mikosch, Modelling Extremal Events (Springer, Heidelberg, 1997) pp. p. 134-138.

${ }^{41}$ M. Sherlock, E. G. Hill, R. G. Evans, S. J. Rose, and W. Rozmus, Phys. Rev. Lett. 113, 255001 (2014).

${ }^{42}$ G. M. Zaslavskii and N. N. Filonenko, Soviet Physics JETP 25, 851 (1968).

${ }^{43}$ D. F. Escande and F. Doveil, Journal of Statistical Physics 26, 257 (1981).

${ }^{44}$ A. J. Lichtenberg and M. A. Lieberman, Regular and Stochastic Motion (Springer, New York, 1983) pp. p. 286-296.

${ }^{45}$ W. L. Kruer and K. Estabrook, Physics of Fluids 28, 430 (1985).

${ }^{46}$ V. A. Vshivkov, N. M. Naumova, F. Pegoraro, and S. V. Bulanov, Physics of Plasmas 5, 2727 (1998), https://doi.org/10.1063/1.872961.

${ }^{47}$ P. Mulser, S. M. Weng, and T. Liseykina, Physics of Plasmas 19, 043301 (2012).

${ }^{48}$ F. Brunel, Phys. Rev. Lett. 59, 52 (1987).

${ }^{49}$ P. Mulser, D. Bauer, and H. Ruhl, Phys. Rev. Lett. 101, 225002 (2008).

${ }^{50}$ B. S. Paradkar, S. I. Krasheninnikov, and F. N. Beg, Physics of Plasmas 19, 060703 (2012).

${ }^{51}$ S. I. Krasheninnikov, Physics of Plasmas 21, 104510 (2014).

${ }^{52}$ B. Bezzerides, S. J. Gitomer, and D. W. Forslund, Phys. Rev. Lett. 44, 651 (1980).

${ }^{53}$ A. Macchi, T. V. Liseikina, S. Tuveri, and S. Veghini, Comptes Rendus Physique 10, 207 (2009), laser acceleration of particles in plasma.

${ }^{54}$ W. Yu, V. Bychenkov, Y. Sentoku, M. Y. Yu, Z. M. Sheng, and K. Mima, Phys. Rev. Lett. 85, $570(2000)$.

${ }^{55}$ B. V. Chirikov, Physics Reports 52, 263 (1979).

${ }^{56}$ A. Bourdier, D. Patin, and E. Lefebvre, Physica D: Nonlinear Phenomena 206, 1 (2005).

${ }^{57}$ D. Patin, E. Lefebvre, A. Bourdier, and E. D’Humières, Laser and Particle Beams 24, 223 (2006).

${ }^{58}$ A. Bourdier, D. Patin, and E. Lefebvre, Laser and Particle Beams 25, 169 (2007).

${ }^{59}$ G. Schmidt, Comments Plasma Phys. Controlled Fusion 7, 87 (1982).

${ }^{60}$ P. W. Smorenburg, J. H. M. Kanters, A. Lassise, G. J. H. Brussaard, L. P. J. Kamp, and O. J. Luiten, Phys. Rev. A 83, 063810 (2011).

${ }^{61}$ We neglect here the possible issue of relativistic reversal considered in Refs. ? ?.

${ }^{62}$ P. L. Kapitza and P. A. M. Dirac, Mathematical Proceedings of the Cambridge Philosophical Society 29, 297-300 (1933). 
${ }^{63}$ P. H. Bucksbaum, D. W. Schumacher, and M. Bashkansky, Phys. Rev. Lett. 61, 1182 (1988).

${ }^{64}$ G. M. Zaslavskii and B. V. Chirikov, Soviet Physics Uspekhi 14, 549 (1972).

${ }^{65}$ A. Lichtenberg, M. Lieberman, and R. Cohen, Physica D: Nonlinear Phenomena 1, 291 (1980).

${ }^{66}$ J. M. Greene, Journal of Mathematical Physics 20, 1183 (1979).

${ }^{67}$ S. V. Coggeshall and J. Meyer-ter Vehn, Journal of Mathematical Physics 33, 3585 (1992).

${ }^{68}$ G. Barenblatt, Scaling, Self-similarity, and Intermediate Asymptotics: Dimensional Analysis and Intermediate Asymptotics, Cambridge Texts in Applied Mathematics (Cambridge University Press, 1996).

${ }^{69}$ M. Raynaud, A. Héron, and J.-C. Adam, Plasma Physics and Controlled Fusion 60, 014021 (2018).

${ }^{70}$ L. M. Wickens, J. E. Allen, and P. T. Rumsby, Phys. Rev. Lett. 41, 243 (1978).

${ }^{71}$ A. Gurevich, D. Anderson, and H. Wilhelmsson, Phys. Rev. Lett. 42, 769 (1979).

${ }^{72}$ M. A. True, J. R. Albritton, and E. A. Williams, The Physics of Fluids 24, 1885 (1981).

${ }^{73}$ V. F. Kovalev, V. Y. Bychenkov, and V. T. Tikhonchuk, Journal of Experimental and Theoretical Physics 95, 226 (2002).

${ }^{74}$ V. F. Kovalev and V. Y. Bychenkov, Phys. Rev. Lett. 90, 185004 (2003).

${ }^{75}$ P. Mora, Phys. Rev. Lett. 90, 185002 (2003).

${ }^{76}$ P. Mora, Phys. Rev. E 72, 056401 (2005).

${ }^{77}$ P. Mora, Physics of Plasmas 12, 112102 (2005).

${ }^{78}$ T. Grismayer and P. Mora, Physics of Plasmas 13, 032103 (2006), https://doi.org/10.1063/1.2178653.

${ }^{79}$ T. Grismayer, P. Mora, J. C. Adam, and A. Héron, Phys. Rev. E 77, 066407 (2008).

${ }^{80}$ A. Diaw and P. Mora, Phys. Rev. E 86, 026403 (2012).

${ }^{81}$ Diaw, A. and Mora, P., EPJ Web of Conferences 59, 17009 (2013).

${ }^{82}$ T. Kiefer and T. Schlegel, Physics of Plasmas 19, 102101 (2012).

${ }^{83}$ D. Bennaceur-Doumaz, D. Bara, E. Benkhelifa, and M. Djebli, Journal of Applied Physics 117, 043303 (2015). 